\title{
The Twin Deficits in OECD Countries: Cointegration Analysis with Regime Shifts ${ }^{1}$
}

\author{
Jalal Siddiki $^{2}$ and Vince Daly ${ }^{3}$
}

\begin{abstract}
We investigate whether government budget deficits and real interest rates have a longrun relationship with the current account of the balance of payments in 23 OECD countries. Such an investigation is of interest since large and persistent budget deficits may impose strains on the foreign exchange markets and are considered by some to be one of the main causes of crises in international financial markets. We permit regime shifts in the cointegration analysis, which extends empirical modelling relative to existing studies. We find that the admission of regime shifts substantially influences the empirical conclusions: we find a long run relationship between budget deficits, real interest rate and current account deficit in 13 out of 23 countries whereas the number of countries with apparent long-run relationships is dramatically reduced when regime shifts are not permitted. We argue that, when structural breaks are taken into account, it seems to be the countries with a more extensive financial infrastructure in which the twin deficits are less likely to be conjoined.
\end{abstract}

Keywords: Twin Deficits, Structural Breaks

JEL Classification: E6, F4

\footnotetext{
${ }^{1}$ We are grateful to colleagues in the Economics Research Seminar programme at Kingston University for their advice and encouragement.

${ }^{2}$ Dr. Jalal U. Siddiki, School of Economics, Kingston University, Penrhyn Road, Kingston, Surrey KT1 2EE, UK. Tel:+44(0)208 547 2000; E-mail: J.Siddiki@kingston.ac.uk

${ }^{3}$ Vincent Daly, Head of Economics, Kingston University, Penrhyn Road, Kingston, Surrey KT1 2EE, UK. Tel:+44(0)208 547 7335; E-mail: V.Daly@kingston.ac.uk
} 


\section{Introduction}

The aim of this paper is to explore the impact of budget deficits and real interest rates on the current accounts of the balance of payments, using cointegration analyses with structural breaks. Variability of current account and budget deficits across timeseries and cross-section dimensions is high in both developed and developing countries (Chinn and Prasad (2003)) and large current account deficits have been considered as one of the main factors causing major currency crashes; see Edwards (2001) for a survey. The notion of current account sustainability has gained policy significance in the context of recent episodes of macroeconomic turbulence (Chinn and Prasad (2003), p. 48). Hence the question of whether or not government budget deficits influence current account deficits in some predictable way has important policy implications.

The existing theoretical and empirical literature dealing with the impact of budget deficits on trade deficits is inconclusive (Ghatak and Ghatak, 1996; Ricciuti, 2003). We also note that most of the empirical studies based on time series data do not incorporate structural breaks in their modelling. To address this gap in the literature, this paper examines the interaction of trade deficits with budget deficits and real interest rates using cointegration analysis in 23 developed countries.

Cointegration implies that the twin deficits and interest rates do not move independently of each other and thus favours the Keynesian perspective - see section 2 below. Tests for cointegration that follow Engle and Granger (1987) - henceforth EG, presume that the cointegrating vector is time-invariant. This is not appropriate if the relationship between the variables of interest experiences a structural break at some date within the sample period. Identifying the existence of such regime shifts is important 
since unacknowledged regime changes might lead to mis-specification bias in model estimation. We therefore apply the approach of Gregory and Hansen (1996) henceforth GH, which permits a structural break.

This paper is organised as follows: in section two, the existing literature is reviewed and the empirical specification of the model is explained. Section three presents the conclusions to be drawn from the empirical results. The methodology is outlined in appendix A. Appendix B collates the detailed statistical results.

\section{Theory and Empirical Model Specification}

There are several channels through which budget deficits may affect trade deficits (Khalid and Guan, 1999, p. 390; Rosenswieg and Tallman, 1993, p. 580). Firstly, in a Mundell-Fleming framework and under flexible exchange rates, an increase in government deficits would induce an upward pressure on real interest rates, causing capital inflows and thereby raising the foreign exchange value of domestic currency, i.e. an appreciation in real exchange rates. The stronger currency in turn reduces net exports, i.e. there is a rise (or fall) in trade deficits (or surpluses). This mechanism is also effective under fixed exchange rate regimes. The Keynesian proposition asserts that the fiscal stimulus under a fixed exchange rate generates higher nominal income, causing an appreciation of domestic currency that worsens the current account balance see Anoruo and Ramchander (1998). The Keynesian absorption theory predicts that a rise in budget deficits increases domestic expenditure and hence an expansion in imports causes current account deficits. Thus, a rise in budget deficits and a resulting increase in the real interest rate increase (reduce) current account deficits (surpluses). 
Finally, a correlation between saving and investment (Feldstein and Horioka, 1980) would also cause budget deficit and current account deficit to move together, supporting the twin deficits hypothesis. The link between fiscal accounts and the external balance can be expressed by rearranging the national income accounting identity:

$$
\left(I_{P}-S_{P}\right)+(G-T)=M-X-N T
$$

where $I_{P}$ is private investment, $S_{P}$ is private saving, $G$ is government spending, $T$ is government revenue, $M$ is imports, $X$ is exports and $N T$ is net current transfers from abroad. This equation states that as long as $\left(I_{P}-S_{P}\right)$ remains stable, changes in fiscal deficits $(G-T)$ will be closely associated with movements in current account deficits $\left(X-M-N_{T}\right)^{4}$.

Contrary to the Mundel-Fleming model, the Ricardian equivalence hypothesis (REH) predicts no causal relationship between trade and budget deficits: shifts between taxes and budget deficits do not have any impact upon the real interest rate, the quantity of investment or the current account balance. A reduction in current taxes will not affect desired national saving, when government spending is held constant and there are no borrowing constraints (Ricciuti, 2003, for a survey). This follows from the argument that a present tax cut is inevitably followed by a future tax increase. Rational response by non-myopic citizens implies that the resulting government deficit does not have any impact upon the economy (Barro, 1974)). A model incorporating Ricardian equivalence would suggest that a substitution of debt for taxes by the government that increases the

\footnotetext{
${ }^{4}$ However, the relationship between fiscal and external deficits may be weakened if increases in government expenditures are associated with reductions of private investment (crowding out effect). This happens when economic agents can anticipate that a current increase in public debt is associated with a future tax increase.
} 
fiscal deficits would be financed by increased private saving, rather than increased net foreign borrowing (trade deficits) (Rosenswieg and Tallman, 1993, p. 582).

The following specification can be used to test whether the current account deficit is independent of the fiscal deficit:

$$
C A G D P=\mu+\beta_{1} B D G D P+\beta_{2} r
$$

We use annual data covering the period 1960 - 2000. CAGDP and BDGDP are, respectively, the current account and budget deficits, as a percentage of GDP, $r$ is the real interest rate (nominal interest rate minus the rate of inflation). The existence of cointegration between these three variables, or between $C A G D P$ and $B D G D P$ alone, is evidence that the two deficits do not move independently, as the REH perspective would have it. Positive values for $\beta_{1}$ and $\beta_{2}$ in the cointegrating vector support the Keynesian perspective.

\section{Empirical Results}

We follow GH in considering the case where the individual series are integrated of order one - I(1). Hence, we preceded our application of the GH procedure with univariate investigation of the constituent series for each country. Using ADF tests ${ }^{5}$ with two lags, we found that almost all of our series behave as $I(1)$ within the estimation period. The unit root null was rejected in 4 of the 69 series at a $10 \%$ significance level. We do not exclude any of these countries since we might expect 6 or 7 false rejections by chance at this significance level, and no country suffered more than

\footnotetext{
${ }^{5}$ Reported more fully in Appendix B.
} 
one such rejection. In seven series the test statistic suggested acceptance of a unit root in the differences, suggesting possible $I(2)$ behaviour. For this exercise we have not excluded the countries in question but note Italy as a country which failed twice in these preliminary diagnostics.

Our main objective has been to discover whether the admission of structural breaks might substantially impact on the empirical support for either of the Keynesian and REH perspectives. Table 1 summarises the evidence ${ }^{6}$ and is read as follows. Results for each country are reported in each of three scenarios: standard cointegration without breaks (SC), a level shift in which only the intercept of the cointegrating vector is permitted to break (LS), and a regime shift in which the slope coefficients of the cointegrating vector may also break (RS). For each of these scenarios the table shows the estimated slope coefficients in equation (2.2) with their OLS t-statistics in parentheses. In the RS case the table also shows the estimated post-break changes to the slopes, with their t-statistics. Shaded cells indicate instances where the test procedure suggests cointegration at an asymptotic test size of either 5\% (superscripted "cc"), or $10 \%$ (superscripted "c").

\begin{tabular}{|c|c|c|c|c|c|c|c|c|}
\hline \multicolumn{1}{|c|}{ Table 1 : cointegration between CAGDP, BDGDP \& $r$} \\
\hline & \multicolumn{3}{|c|}{ SC } & \multicolumn{3}{c|}{ LS } & \multicolumn{4}{c|}{ RS } \\
\hline & $\beta_{1}$ & $\beta_{2}$ & $\beta_{1}$ & $\beta_{2}$ & $\beta_{1}$ & $\beta_{2}$ & $\beta_{3}$ & $\beta_{4}$ \\
\hline Austria & $0.04^{\mathrm{c}}$ & $0.18^{\mathrm{c}}$ & $0.31^{\mathrm{cc}}$ & $0.06^{\mathrm{cc}}$ & $0.47^{\mathrm{c}}$ & $0.03^{\mathrm{c}}$ & $-0.70^{\mathrm{c}}$ & $0.05^{\mathrm{c}}$ \\
& $(0.33)$ & $(1.73)$ & $(2.06)$ & $(0.58)$ & $(2.72)$ & $(0.25)$ & $(-1.88)$ & $(0.24)$ \\
\hline Australia & $0.19^{\mathrm{cc}}$ & $-0.24^{\mathrm{cc}}$ & $0.04^{\mathrm{cc}}$ & $-0.19^{\mathrm{cc}}$ & $0.19^{\mathrm{cc}}$ & $-0.45^{\mathrm{cc}}$ & $-0.26^{\mathrm{cc}}$ & $0.30^{\mathrm{cc}}$ \\
& $(1.28)$ & $(-3.82)$ & $(0.26)$ & $(-3.00)$ & $(0.64)$ & $(-2.53)$ & $(-0.75)$ & $(1.63)$ \\
\hline Belgium & -0.03 & -0.06 & 0.01 & -0.18 & -0.08 & 0.19 & 0.60 & -0.17 \\
& $(-0.88)$ & $(-0.50)$ & $(0.47)$ & $(-2.21)$ & $(-1.75)$ & $(1.02)$ & $(4.40)$ & $(-0.64)$ \\
\hline Canada & -0.01 & 0.27 & 0.13 & -0.01 & 0.26 & -0.03 & -0.17 & 0.01 \\
& $(-0.07)$ & $(2.66)$ & $(1.63)$ & $(-0.10)$ & $(1.52)$ & $(-0.18)$ & $(-0.86)$ & $(0.03)$ \\
\hline Denmark & -0.06 & 0.29 & $-0.003^{\mathrm{cc}}$ & $-0.02^{\mathrm{cc}}$ & $0.16^{\mathrm{cc}}$ & $-0.01^{\mathrm{cc}}$ & $-0.96^{\mathrm{cc}}$ & $-0.04^{\mathrm{cc}}$ \\
& $(-0.54)$ & $(2.57)$ & $(-0.04)$ & $(-0.17)$ & $(1.59)$ & $(-0.08)$ & $(-4.58)$ & $(-0.17)$ \\
\hline
\end{tabular}

\footnotetext{
${ }^{6}$ Additional detail is provided in Appendix B.
} 


\begin{tabular}{|c|c|c|c|c|c|c|c|c|}
\hline & \multicolumn{2}{|c|}{$\mathrm{SC}$} & \multicolumn{2}{|c|}{ LS } & \multicolumn{4}{|c|}{$\mathrm{RS}$} \\
\hline & $\beta_{1}$ & $\beta_{2}$ & $\beta_{1}$ & $\beta_{2}$ & $\beta_{1}$ & $\beta_{2}$ & $\beta_{3}$ & $\beta_{4}$ \\
\hline Finland & $\begin{array}{c}-0.02 \\
(-0.16)\end{array}$ & $\begin{array}{c}0.23 \\
(1.66)\end{array}$ & $\begin{array}{c}0.46 \\
(3.53)\end{array}$ & $\begin{array}{c}0.14 \\
(1.34)\end{array}$ & $\begin{array}{c}-0.27 \\
(-0.85)\end{array}$ & $\begin{array}{c}0.24 \\
(1.85)\end{array}$ & $\begin{array}{c}0.04 \\
(0.09)\end{array}$ & $\begin{array}{c}-2.54 \\
(-2.51)\end{array}$ \\
\hline France & $\begin{array}{c}-0.08 \\
(-0.09) \\
\end{array}$ & $\begin{array}{c}0.10 \\
(1.67)\end{array}$ & $\begin{array}{l}0.05^{\mathrm{cc}} \\
(0.45)\end{array}$ & $\begin{array}{l}0.06^{\mathrm{cc}} \\
(1.06) \\
\end{array}$ & $\begin{array}{c}0.12^{\mathrm{c}} \\
(0.95)\end{array}$ & $\begin{array}{c}0.07^{c} \\
(1.09)\end{array}$ & $\begin{array}{c}-0.16^{\mathrm{C}} \\
(-0.51) \\
\end{array}$ & $\begin{array}{c}0.32^{\mathrm{c}} \\
(0.37)\end{array}$ \\
\hline Germany & $\begin{array}{c}0.21 \\
(0.86)\end{array}$ & $\begin{array}{c}0.14 \\
(0.86)\end{array}$ & $\begin{array}{c}0.05 \\
(0.23)\end{array}$ & $\begin{array}{c}0.17 \\
(1.13)\end{array}$ & $\begin{array}{c}0.09 \\
(0.37)\end{array}$ & $\begin{array}{c}-0.12 \\
(-0.56)\end{array}$ & $\begin{array}{c}2.20 \\
(3.20)\end{array}$ & $\begin{array}{c}0.44 \\
(1.22)\end{array}$ \\
\hline Greece & $\begin{array}{l}0.09^{\mathrm{cc}} \\
(1.88)\end{array}$ & $\begin{array}{l}0.20^{c c} \\
(4.94)\end{array}$ & $\begin{array}{l}-0.01^{\mathrm{cc}} \\
(-0.14)\end{array}$ & $\begin{array}{l}0.21^{\mathrm{cc}} \\
(5.25)\end{array}$ & $\begin{array}{l}0.38^{\mathrm{cc}} \\
(4.28)\end{array}$ & $\begin{array}{l}0.12^{\mathrm{cc}} \\
(2.41)\end{array}$ & $\begin{array}{l}-0.47^{\mathrm{cc}} \\
(-3.78)\end{array}$ & $\begin{array}{l}0.05^{\mathrm{cc}} \\
(0.36)\end{array}$ \\
\hline Iceland & $\begin{array}{l}0.71^{\mathrm{cc}} \\
(2.01)\end{array}$ & $\begin{array}{l}0.03^{\mathrm{cc}} \\
(1.03)\end{array}$ & $\begin{array}{l}0.82^{\mathrm{cC}} \\
(2.22)\end{array}$ & $\begin{array}{l}0.02^{\mathrm{cc}} \\
(0.41)\end{array}$ & $\begin{array}{l}1.37^{\mathrm{cC}} \\
(3.25)\end{array}$ & $\begin{array}{l}0.01^{\mathrm{cc}} \\
(0.12)\end{array}$ & $\begin{array}{c}-1.59 \\
(-2.12)\end{array}$ & $\begin{array}{c}-0.11 \\
(-0.92)\end{array}$ \\
\hline Ireland & $\begin{array}{c}1.59^{c} \\
(3.24)\end{array}$ & $\begin{array}{l}1.19^{\mathrm{C}} \\
(2.21)\end{array}$ & $\begin{array}{l}1.71^{\mathrm{cc}} \\
(3.63)\end{array}$ & $\begin{array}{l}1.14^{\mathrm{cc}} \\
(2.22)\end{array}$ & $\begin{array}{l}0.68^{\mathrm{cc}} \\
(0.52)\end{array}$ & $\begin{array}{l}0.34^{\mathrm{cc}} \\
(0.38)\end{array}$ & $\begin{array}{l}1.02^{\mathrm{cc}} \\
(0.72)\end{array}$ & $\begin{array}{l}1.07^{\mathrm{cc}} \\
(0.87)\end{array}$ \\
\hline Italy & $\begin{array}{c}0.25^{\mathrm{c}} \\
(3.60) \\
\end{array}$ & $\begin{array}{c}0.09^{c} \\
(1.38) \\
\end{array}$ & $\begin{array}{c}0.31 \\
(3.77) \\
\end{array}$ & $\begin{array}{c}0.08 \\
(1.30) \\
\end{array}$ & $\begin{array}{c}-0.14 \\
(-0.61) \\
\end{array}$ & $\begin{array}{c}0.59 \\
(2.44) \\
\end{array}$ & $\begin{array}{c}0.37 \\
(1.49) \\
\end{array}$ & $\begin{array}{c}-0.52 \\
(-2.09) \\
\end{array}$ \\
\hline Japan & $\begin{array}{c}0.17 \\
(1.87)\end{array}$ & $\begin{array}{c}0.22 \\
(3.28)\end{array}$ & $\begin{array}{c}0.11^{\mathrm{C}} \\
(1.18)\end{array}$ & $\begin{array}{c}0.10^{c} \\
(1.17)\end{array}$ & $\begin{array}{c}0.11 \\
(0.79)\end{array}$ & $\begin{array}{c}0.13 \\
(1.37)\end{array}$ & $\begin{array}{c}0.11 \\
(0.61)\end{array}$ & $\begin{array}{c}0.53 \\
(1.66)\end{array}$ \\
\hline Luxembourg & $\begin{array}{c}0.61 \\
(1.92)\end{array}$ & $\begin{array}{c}-0.52 \\
(-1.31)\end{array}$ & $\begin{array}{c}0.95 \\
(3.12)\end{array}$ & $\begin{array}{c}-0.94 \\
(-2.48)\end{array}$ & $\begin{array}{c}2.97^{\mathrm{C}} \\
(3.35)\end{array}$ & $\begin{array}{l}-1.40^{\mathrm{C}} \\
(-1.97)\end{array}$ & $\begin{array}{l}-2.60^{c} \\
(-2.78)\end{array}$ & $\begin{array}{c}0.71^{\mathrm{C}} \\
(0.66)\end{array}$ \\
\hline Netherlands & $\begin{array}{c}-0.06 \\
(-0.34)\end{array}$ & $\begin{array}{c}0.07 \\
(0.53)\end{array}$ & $\begin{array}{l}0.25^{\mathrm{cc}} \\
(2.09)\end{array}$ & $\begin{array}{l}-0.32^{\mathrm{cc}} \\
(-3.08)\end{array}$ & $\begin{array}{c}0.45 \\
(2.01)\end{array}$ & $\begin{array}{c}-0.28 \\
(-2.23)\end{array}$ & $\begin{array}{c}-0.27 \\
(-1.01)\end{array}$ & $\begin{array}{c}-0.11 \\
(-0.47)\end{array}$ \\
\hline New Zealand & $\begin{array}{c}0.10 \\
(0.64)\end{array}$ & $\begin{array}{c}0.02 \\
(0.17)\end{array}$ & $\begin{array}{l}0.37^{\mathrm{cC}} \\
(2.97)\end{array}$ & $\begin{array}{l}0.36^{\mathrm{cc}} \\
(3.26)\end{array}$ & $\begin{array}{l}0.82^{\mathrm{cc}} \\
(2.46)\end{array}$ & $\begin{array}{l}0.28^{\mathrm{cc}} \\
(1.58)\end{array}$ & $\begin{array}{l}-0.72^{\mathrm{cc}} \\
(-2.03)\end{array}$ & $\begin{array}{l}-0.30^{\mathrm{cc}} \\
(-1.41)\end{array}$ \\
\hline Norway & $\begin{array}{c}0.59 \\
(2.43) \\
\end{array}$ & $\begin{array}{c}0.39 \\
(2.63)\end{array}$ & $\begin{array}{c}0.78^{\mathrm{c}} \\
(3.54) \\
\end{array}$ & $\begin{array}{c}0.08^{\mathrm{c}} \\
(0.52) \\
\end{array}$ & $\begin{array}{l}1.27^{\mathrm{cC}} \\
(4.60) \\
\end{array}$ & $\begin{array}{l}0.43^{\mathrm{cc}} \\
(1.97)\end{array}$ & $\begin{array}{l}-1.78^{\mathrm{cc}} \\
(-4.26)\end{array}$ & $\begin{array}{l}-1.69^{\mathrm{cc}} \\
(-2.73)\end{array}$ \\
\hline Portugal & $\begin{array}{c}0.23 \\
(1.20)\end{array}$ & $\begin{array}{c}0.19 \\
(1.74)\end{array}$ & $\begin{array}{l}0.45^{\mathrm{cC}} \\
(2.19)\end{array}$ & $\begin{array}{l}0.35^{\mathrm{cC}} \\
(2.83)\end{array}$ & $\begin{array}{c}0.59 \\
(2.96)\end{array}$ & $\begin{array}{c}0.37 \\
(2.95)\end{array}$ & $\begin{array}{c}-1.60 \\
(-2.40)\end{array}$ & $\begin{array}{c}-0.06 \\
(-0.17)\end{array}$ \\
\hline Spain & $\begin{array}{l}-0.08^{\mathrm{cc}} \\
(-0.61)\end{array}$ & $\begin{array}{l}-0.01^{\text {cc }} \\
(0.25)\end{array}$ & $\begin{array}{l}-0.20^{\mathrm{cc}} \\
(-1.31) \\
\end{array}$ & $\begin{array}{c}-0.004^{\mathrm{cc}} \\
(-0.07)\end{array}$ & $\begin{array}{c}-0.004^{\mathrm{cc}} \\
(-0.03)\end{array}$ & $\begin{array}{l}0.07^{\mathrm{cc}} \\
(1.36)\end{array}$ & $\begin{array}{l}-0.10^{\mathrm{cc}} \\
(-0.33)\end{array}$ & $\begin{array}{l}-0.65^{\mathrm{cc}} \\
(-2.85)\end{array}$ \\
\hline Sweden & $\begin{array}{c}0.14 \\
(2.06)\end{array}$ & $\begin{array}{c}0.14 \\
(1.18)\end{array}$ & $\begin{array}{c}0.16 \\
(2.43)\end{array}$ & $\begin{array}{c}0.02 \\
(0.22)\end{array}$ & $\begin{array}{c}0.14 \\
(1.58)\end{array}$ & $\begin{array}{c}0.01 \\
(0.04)\end{array}$ & $\begin{array}{c}0.07 \\
(0.54)\end{array}$ & $\begin{array}{c}-0.02 \\
(-0.07)\end{array}$ \\
\hline Switzerland & $\begin{array}{c}-1.85 \\
(-3.15)\end{array}$ & $\begin{array}{c}1.21 \\
(4.40)\end{array}$ & $\begin{array}{c}-1.22 \\
(-2.57)\end{array}$ & $\begin{array}{c}0.90 \\
(3.97)\end{array}$ & $\begin{array}{c}-1.97 \\
(-2.97)\end{array}$ & $\begin{array}{c}1.01 \\
(3.85)\end{array}$ & $\begin{array}{c}2.72 \\
(2.60)\end{array}$ & $\begin{array}{c}-1.26 \\
(-1.45)\end{array}$ \\
\hline United Kingdom & $\begin{array}{c}-0.06 \\
(-0.49)\end{array}$ & $\begin{array}{c}-0.04 \\
(-0.58)\end{array}$ & $\begin{array}{c}-0.10 \\
(-0.99)\end{array}$ & $\begin{array}{c}0.09 \\
(1.50)\end{array}$ & $\begin{array}{c}0.07 \\
(0.55)\end{array}$ & $\begin{array}{c}0.07 \\
(1.10)\end{array}$ & $\begin{array}{c}-0.34 \\
(-1.80)\end{array}$ & $\begin{array}{c}-0.09 \\
(-0.44)\end{array}$ \\
\hline USA & $\begin{array}{c}0.02 \\
(0.27)\end{array}$ & $\begin{array}{c}-0.41 \\
(-3.68)\end{array}$ & $\begin{array}{c}-0.15 \\
(-1.82)\end{array}$ & $\begin{array}{c}-0.23 \\
(-2.52)\end{array}$ & $\begin{array}{c}-0.02 \\
(-0.14)\end{array}$ & $\begin{array}{c}0.02 \\
(0.13)\end{array}$ & $\begin{array}{c}-0.20 \\
(-1.03)\end{array}$ & $\begin{array}{c}-0.48 \\
(-2.81)\end{array}$ \\
\hline
\end{tabular}

It is evident that the conclusions with regards to cointegration are substantially altered by permitting structural breaks. On balance, there is a noticeable tendency to discover cointegration more often than when structural breaks are not permitted. We find a long run relationship between budget deficits, real interest rate and current account deficit in 14 out of 23 countries when a break is permitted but in only 7 countries using cointegration analysis without breaks. This suggests that earlier studies which did not 
allow for structural breaks may have been methodologically biased in favour of supporting the REH perspective.

As to whether the Keynesian perspective is positively supported, we can note that, for the (SC) model without breaks, Spain is the only country for which we discover a cointegrating vector where the sign of the coefficient on BDGDP is contrary to (Keynesian) expectation. This is also true for the pre-break sub-sample of the regime shift (RS) scenario but the post-break sub-sample is much less supportive of the Keynesian perspective.

Although this research is preliminary, it is tempting to offer some explanation as to why the competing perspectives - Keynesian vs. REH, might each find some evidence to favour them. Using the least restrictive (RS) scenario as a basis for discussion, we can note that countries which have an established reputation as international financial centres are more likely to provide evidence, i.e. noncointegration, in favour of the REH perspective. We might therefore posit the hypothesis that a more developed financial infrastructure makes it more likely that what is logically rational behaviour in the theory of Ricardian Equivalence is also feasible action in the real world. It is also noteworthy that, where cointegration is detected in the RS scenario, the signs of the estimated coefficients are less supportive of the Keynesian perspective in the post-break sub-sample, which may be grounds for speculating that a strengthening of financial infrastructure throughout these OECD countries has rendered the Keynesian case generally less compelling. 


\section{References}

Anoruo, E. and Ramchander, S. (1998) Current Account and Fiscal Deficits: Evidence from Five Developing Economies of Asia, Journal of Asian Economics, 9 (3), 487- 501.

Barro, R. (1974) Are government bonds net wealth?, Journal of Political Economy, 82, $1095-1117$.

Chinn, M. D. and Prasad, E. S. (2003) Medium-term determinants of current accounts in industrial and developing countries: an empirical exploration, Journal of International Economics, 59 (1), 47-76

Edwards, S. (2001) Does the Current Account Matter? NBER Working Papers, 8275

Engle, R. F. and Granger, C.W.J. (1987) Cointegration and Error Correction: Representation, Estimation and Testing, Econometrica, 55 (2), 251-76.

Feldstein, M. and Horioka, C. (1980) Domestic Saving and International Capital Flows, Economic Journal, 90, 314-329.

Ghatak, A. and Ghatak, S. (1996) Budgetary deficits and Ricardian equivalence: The case of India, 1950-1986, Journal of Public Economics, 60 (2), 267-82.

Gregory, A. W. and Hansen, B.E. (1996) Residual Based Tests for Cointegration in Models with Regime Shifts, Journal of Econometrics, 70 (1), 99-126.

Khalid, A. M. and Guan, T. W. (1999) Casuality Tests of Budget and Current Account Deficits: Cross-Country Comparisons, Empirical Economics, 24 (3), 389-402.

Ricciuti, R. (2003) Assessing Ricardian Equivalence, Journal of Economic Surveys, 17 (1), $55-78$.

Rosenswieg, J. A. and Tallman, E. W. (1993) Fiscal Policy and Trade Adjustment: Are the Deficits Really Twins? Economic Inquiry, 31 (4), 580-594. 


\section{Appendix A: Methodology}

Gregory and Hansen (1996) (GH) modify the Engle-Granger (1987) (EG) approach to testing for the existence of a single cointegrating relationship by allowing that the coefficients of this equation may undergo a structural break. The date of the break is not specified beforehand; it is estimated within the GH procedure by considering all dates within the sample (except those too close to the sample end-points) as candidates for a structural break and selecting from these the one that produces the most convincing evidence of cointegration. To decide whether this evidence is statistically significant, the GH procedure replaces the critical values appropriate to the EG approach with critical values arrived at by Monte Carlo simulation of the modified procedure.

In our investigation, equation 2.2 provides the vehicle for EG tests for cointegration. We call it the "standard cointegration" model (SC), viz:

$$
\begin{array}{ll} 
& C A G D P_{t}=\mu_{1}+\beta_{1} B D G D P_{t}+\beta_{2} r_{t}+\varepsilon_{t} \\
\text { Model SC: } & t=1,2 \cdots n
\end{array}
$$

The GH procedure requires that we modify this equation by introducing a dummy variable to indicate the break date, which is

$$
\varphi_{1 t}=\left\{\begin{array}{lll}
0 & \text { if } & t \leq[n \tau] \\
1 & \text { if } & t>[n \tau]
\end{array} \quad 0.15 \leq \tau \leq 0.85\right.
$$

(Here $[x]$ indicates the integer part of $x$.) This dummy variable can be used to model a variety of forms of structural break. We consider two of these: a level shift (LS) and a regime shift (RS) of the cointegrating equation: 
$\begin{array}{ll} & C A G D P_{t}=\mu_{1}+\mu_{2} \varphi_{1 t}+\beta_{1} B D G D P_{t}+\beta_{2} r_{t}+\varepsilon_{t}\end{array}$

The regime shift model allows for the possibility that the slope coefficients of the cointegrating equation break at the same date:

$$
\begin{gathered}
\text { CAGDP }=\mu_{1}+\beta_{1} B D G D P_{t}+\beta_{2} r_{t} \\
\quad+\mu_{2} \varphi_{1 t}+\beta_{3} \varphi_{1 t} B D G D P_{t}+\beta_{4} \varphi_{1 t} r+\varepsilon_{t} \\
\text { Model RS: } \quad \text { A.4 } \\
t=1,2 \cdots n
\end{gathered}
$$

The GH procedure consists of applying one of these models sequentially for all break dates within the permitted range of $[0.15 n] \leq t \leq[0.85 n]$ and, for each such break date, computing a statistic to test the null of no cointegration. If the most extreme value thus obtained for the test statistic exceeds the GH critical value then we reject the null in favour of "cointegration with a structural break". 


\section{Appendix B: Statistical Results}

Table B1: Unit Root Tests

\begin{tabular}{|c|c|c|c|c|c|c|c|c|}
\hline & & & \multirow{2}{*}{\multicolumn{6}{|c|}{ Variables }} \\
\hline \multirow[t]{3}{*}{ Country } & \multicolumn{2}{|c|}{ Sample Period } & & & & & & \\
\hline & & & \multicolumn{3}{|c|}{ levels } & \multicolumn{3}{|c|}{ First Difference } \\
\hline & & & & & & & & \\
\hline & Begin & End & bdgdp & cagdp & $r$ & bdgdp & cagdp & $r$ \\
\hline Austria & 1960 & 1994 & -1.202 & -2.295 & -1.903 & $-4.346^{* *}$ & $-4.495^{* *}$ & -2.689 \\
\hline Australia & 1960 & 1997 & -3.079 * & -2.020 & -1.463 & $-3.891^{\star \star}$ & $-4.725^{* \star}$ & $-3.743^{\star *}$ \\
\hline Belgium & 1960 & 1998 & -1.493 & -1.942 & -2.167 & $-3.395^{*}$ & $-4.951^{\star *}$ & -3.460 * \\
\hline Canada & 1960 & 2000 & -1.564 & -1.384 & -2.349 & -3.669 ** & $-3.371^{*}$ & $-6.495^{* *}$ \\
\hline Denmark & 1960 & 1999 & $-3.507^{* *}$ & -0.957 & -2.147 & $-4.094^{* *}$ & $-3.307^{*}$ & $-4.535^{* *}$ \\
\hline Finland & 1960 & 1998 & -2.641 & -1.816 & -2.304 & $-3.481^{*}$ & $-4.017^{* *}$ & $-4.827^{* *}$ \\
\hline France & 1960 & 1997 & -1.787 & -2.022 & -1.606 & $-4.137^{* *}$ & $-3.832^{* *}$ & $-5.728^{* *}$ \\
\hline Germany & 1960 & 1998 & -2.354 & -2.168 & -2.265 & $-4.437^{* *}$ & $-3.115^{*}$ & $-4.353^{* *}$ \\
\hline Greece & 1961 & 1999 & -1.456 & -2.955 & -1.418 & $-5.336^{* *}$ & $-6.307^{* *}$ & $-4.152^{* *}$ \\
\hline Iceland & 1960 & 2000 & -2.129 & -2.696 & -1.744 & -3.192 * & $-4.138^{* *}$ & -3.950 ** \\
\hline Ireland & 1960 & 1999 & -0.655 & -1.929 & -2.037 & $-3.409^{*}$ & $-4.787^{* \star}$ & $-4.733^{* \star}$ \\
\hline Italy & 1960 & 1998 & -1.022 & -2.306 & -1.508 & -2.300 & $-4.143^{* *}$ & -2.949 \\
\hline Japan & 1970 & 1993 & -1.155 & -1.704 & -1.366 & -1.269 & $-3.040^{*}$ & $-3.110^{*}$ \\
\hline Luxembourg & 1973 & 1997 & -2.634 & -1.592 & -1.855 & $-3.557^{* \star}$ & $-3.278^{*}$ & $-4.084^{* *}$ \\
\hline Netherlands & 1960 & 1998 & -1.964 & -2.457 & -1.880 & $-4.083^{* *}$ & $-3.702^{* *}$ & $-3.756^{* *}$ \\
\hline New Zealand & 1960 & 2000 & -1.316 & -2.471 & -1.519 & -2.610 & $-4.671^{* *}$ & -3.996 ** \\
\hline Norway & 1960 & 1998 & $-3.191^{*}$ & -2.682 & -1.067 & $-4.741^{\star *}$ & $-4.193^{* *}$ & $-4.304^{* *}$ \\
\hline Portugal & 1970 & 1998 & -1.342 & -2.865 & -1.330 & -2.520 & $-4.187^{* *}$ & $-3.045^{*}$ \\
\hline Spain & 1962 & 1998 & -2.480 & $-4.881^{* *}$ & -1.678 & -2.110 & $-4.818^{* *}$ & $-3.718^{* *}$ \\
\hline Sweden & 1960 & 2000 & -2.944 & -1.748 & -1.649 & $-3.107^{*}$ & $-3.317^{*}$ & $-4.887^{* *}$ \\
\hline Switzerland & 1960 & 2000 & -2.502 & -0.634 & -2.465 & -4.859 ** & $-3.891^{* *}$ & -4.256 ** \\
\hline UK & 1960 & 1999 & -2.713 & -2.493 & -1.814 & $-3.342^{*}$ & -4.090 ** & $-4.462^{* *}$ \\
\hline USA & 1960 & 2000 & -1.053 & -1.079 & -2.361 & -3.691 ** & $-3.098^{*}$ & $-4.675^{* *}$ \\
\hline${ }^{*}$ and ${ }^{* *}$ imply & evel of & ificanc & $10 \%$ anc & respect & The 5\% & $10 \%$ critic & lues are & roximately \\
\hline & & & & & & & & \\
\hline
\end{tabular}


Table B2 - results of GH procedure

\begin{tabular}{|c|c|c|c|c|c|c|c|c|c|c|}
\hline $\begin{array}{c}\text { Country, sample periods } \\
\text { And model types }\end{array}$ & $\mu_{1}$ & $\mu_{1} \varphi_{1 \tau}$ & $\beta_{1}$ & $\beta_{2}$ & $\beta_{3} \varphi_{1 \tau}$ & $\beta_{4} \varphi_{1 \tau}$ & $\begin{array}{l}\text { Break } \\
\text { year }\end{array}$ & ADF & $\mathrm{R}^{2}$ & DW \\
\hline \multicolumn{11}{|c|}{ Austria:1960-1994, one regressor } \\
\hline SC: $C A G D P=f(C, B D G D P)$ & $\begin{array}{c}-0.63 \\
(-1.56)\end{array}$ & & $\begin{array}{l}0.003 \\
(0.02)\end{array}$ & & & & & -3.30 & 0.000 & 0.99 \\
\hline LS: CAGDP=f(C, BDGDP) & $\begin{array}{c}-0.19 \\
(-0.49) \\
\end{array}$ & $\begin{array}{l}1.58^{\star *} \\
(3.04)\end{array}$ & $\begin{array}{l}0.34^{*} \\
(2.69)\end{array}$ & & & & 1981 & $-5.10^{*}$ & 0.224 & 1.58 \\
\hline RS: CAGDP=f(C, BDGDP) & $\begin{array}{c}0.11 \\
(0.27) \\
\end{array}$ & $\begin{array}{c}-1.31 \\
(-0.83)\end{array}$ & $\begin{array}{l}0.47^{* *} \\
(2.98)\end{array}$ & & $\begin{array}{c}-0.69 \\
(-1.93) \\
\end{array}$ & & 1981 & $-5.16^{*}$ & 0.31 & 1.59 \\
\hline \multicolumn{11}{|c|}{ Austria:1960-1994: Two regressors } \\
\hline SC: $C A G D P=f(C, B D G D P, r)$ & $\begin{array}{c}-0.65 \\
(-1.66) \\
\end{array}$ & & $\begin{array}{c}0.04 \\
(0.33) \\
\end{array}$ & $\begin{array}{c}0.18 \\
(1.73) \\
\end{array}$ & & & & $-3.91^{*}$ & 0.09 & 1.21 \\
\hline LS: CAGDP=f(C, BDGDP, r) & $\begin{array}{c}-0.24 \\
(-0.60) \\
\end{array}$ & $\begin{array}{c}1.43^{*} \\
(2.44)\end{array}$ & $\begin{array}{l}0.31^{*} \\
(2.06)\end{array}$ & $\begin{array}{c}0.06 \\
(0.58) \\
\end{array}$ & & & 1981 & $-5.10^{*}$ & 0.23 & 1.60 \\
\hline RS: $C A G D P=f(C, B D G D P, r)$ & $\begin{array}{c}0.08 \\
(0.18)\end{array}$ & $\begin{array}{c}-1.53 \\
(-0.91) \\
\end{array}$ & $\begin{array}{l}0.47^{*} \\
(2.72)\end{array}$ & $\begin{array}{c}0.03 \\
(0.25) \\
\end{array}$ & $\begin{array}{l}-0.70 \\
(-1.88) \\
\end{array}$ & $\begin{array}{l}0.05 \\
(0.24) \\
\end{array}$ & 1981 & $-5.35^{*}$ & 0.31 & 1.62 \\
\hline \multicolumn{11}{|c|}{ Australia:1960-1997: One regressor } \\
\hline SC: $\mathrm{CAGDP}=\mathrm{f}(\mathrm{C}, \mathrm{BDGDP})$ & $\begin{array}{l}-2.95^{\star *} \\
(-8.79)\end{array}$ & & $\begin{array}{c}0.04 \\
(0.21)\end{array}$ & & & & & -3.57 & 0.001 & 0.96 \\
\hline LS: CAGDP=f(C, BDGDP) & $\begin{array}{l}-2.10^{* *} \\
(-5.70)\end{array}$ & $\begin{array}{c}-1.76^{\star *} \\
(-3.71)\end{array}$ & $\begin{array}{l}-0.03 \\
(-0.22)\end{array}$ & & & & 1978 & $-4.92^{\star \star}$ & 0.28 & 1.31 \\
\hline RS: CAGDP=f(C, BDGDP) & $\begin{array}{l}-2.00^{\star *} \\
(-4.98) \\
\end{array}$ & $\begin{array}{l}-1.97^{\star *} \\
(-3.39) \\
\end{array}$ & $\begin{array}{c}0.07 \\
(0.33) \\
\end{array}$ & & $\begin{array}{c}-0.19 \\
(-0.64) \\
\end{array}$ & & 1978 & $-5.05^{* *}$ & 0.29 & 1.33 \\
\hline \multicolumn{11}{|c|}{ Australia:1960-1997: Two regressors } \\
\hline SC: $C A G D P=f(C, B D G D P, r)$ & $\begin{array}{l}-2.08^{* *} \\
(-5.72) \\
\end{array}$ & & $\begin{array}{c}0.19 \\
(1.28)\end{array}$ & $\begin{array}{l}-0.24^{* *} \\
(-3.82) \\
\end{array}$ & & & & $-4.94^{* *}$ & 0.30 & 1.45 \\
\hline LS: CAGDP=f(C, BDGDP, r) & $\begin{array}{l}-1.66^{\star *} \\
(-4.22) \\
\end{array}$ & $\begin{array}{c}-1.18 \\
\left(-2.28^{*}\right)\end{array}$ & $\begin{array}{c}0.04 \\
(0.26) \\
\end{array}$ & $\begin{array}{l}-0.19^{*} \\
(-3.00)\end{array}$ & & & 1974 & $-5.51^{* *}$ & 0.39 & 1.40 \\
\hline RS: CAGDP=f(C, BDGDP, $r)$ & $\begin{array}{l}-1.14^{*} \\
(-2.35) \\
\end{array}$ & $\begin{array}{l}-2.04^{\star *} \\
(-2.86)\end{array}$ & $\begin{array}{c}0.19 \\
(0.64)\end{array}$ & $\begin{array}{l}-0.45^{*} \\
(-2.53)\end{array}$ & $\begin{array}{l}-0.26 \\
(-0.75) \\
\end{array}$ & $\begin{array}{c}0.30 \\
(1.63)\end{array}$ & 1974 & $-5.70^{*}$ & 0.44 & 1.46 \\
\hline \multicolumn{11}{|c|}{ Belgium: 1960 - 1998: One regressor } \\
\hline SC: $\mathrm{CAGDP}=\mathrm{f}(\mathrm{C}, \mathrm{BDGDP})$ & $\begin{array}{c}0.08 \\
(0.25)\end{array}$ & & $\begin{array}{c}-0.02 \\
(-0.79)\end{array}$ & & & & & -0.98 & 0.02 & 0.17 \\
\hline LS: CAGDP=f(C, BDGDP) & $\begin{array}{l}-0.98^{\star *} \\
(-3.25) \\
\end{array}$ & $\begin{array}{l}3.39^{\star *} \\
(6.11)\end{array}$ & $\begin{array}{c}0.02 \\
(0.79) \\
\end{array}$ & & & & 1987 & -3.94 & 0.52 & 0.57 \\
\hline RS: $C A G D P=f(C, B D G D P)$ & $\begin{array}{l}-1.10^{* *} \\
(-3.88) \\
\end{array}$ & $\begin{array}{l}5.05^{\star *} \\
(4.97)\end{array}$ & $\begin{array}{c}0.02 \\
(1.04)\end{array}$ & & $\begin{array}{c}0.30 \\
(1.72)\end{array}$ & & 1986 & -3.98 & 0.60 & 0.46 \\
\hline \multicolumn{11}{|c|}{ Belgium: 1960 - 1998: Two regressors } \\
\hline SC: $C A G D P=f(C, B D G D P, r)$ & $\begin{array}{c}0.25 \\
(0.53)\end{array}$ & & $\begin{array}{c}-0.03 \\
(-0.88)\end{array}$ & $\begin{array}{c}-0.06 \\
(-0.50)\end{array}$ & & & & -0.99 & 0.02 & 0.17 \\
\hline LS: CAGDP=f(C, BDGDP, $r)$ & $\begin{array}{c}-0.56 \\
(-1.62)\end{array}$ & $\begin{array}{l}3.65^{\star *} \\
(6.75)\end{array}$ & $\begin{array}{c}0.01 \\
(0.47)\end{array}$ & $\begin{array}{c}-0.18 \\
(-2.21)\end{array}$ & & & 1987 & -3.77 & 0.58 & 0.75 \\
\hline RS: CAGDP=f(C, BDGDP, r) & $\begin{array}{c}0.27 \\
(0.49)\end{array}$ & $\begin{array}{l}3.73^{\star *} \\
(3.37)\end{array}$ & $\begin{array}{c}-0.08 \\
(-1.75)\end{array}$ & $\begin{array}{c}0.19 \\
(1.02)\end{array}$ & $\begin{array}{l}0.60^{\star *} \\
(4.40)\end{array}$ & $\begin{array}{c}-0.17 \\
(-0.64)\end{array}$ & 1977 & -3.94 & 0.43 & 0.44 \\
\hline
\end{tabular}


Table B2 continued

\begin{tabular}{|c|c|c|c|c|c|c|c|c|c|c|}
\hline $\begin{array}{l}\text { Country, sample periods } \\
\text { And model types }\end{array}$ & $\mu_{1}$ & $\mu_{1} \varphi_{1 \tau}$ & $\beta_{1}$ & $\beta_{2}$ & $\beta_{3} \varphi_{1 \tau}$ & $\beta_{4} \varphi_{1 \tau}$ & $\begin{array}{c}\text { Break } \\
\text { year }\end{array}$ & ADF & $\mathrm{R}^{2}$ & DW \\
\hline \multicolumn{11}{|c|}{ Canada:1960-2000: One regressor } \\
\hline SC: $C A G D P=f(C, B D G D P)$ & $\begin{array}{c}-0.27 \\
(-0.69)\end{array}$ & & $\begin{array}{c}-0.09 \\
(-0.81)\end{array}$ & & & & & -1.25 & 0.02 & 0.28 \\
\hline LS: CAGDP=f(C, BDGDP) & $\begin{array}{c}-0.98 \\
(-3.59)\end{array}$ & $\begin{array}{l}2.58^{* \star} \\
(7.37)\end{array}$ & $\begin{array}{c}0.13 \\
(1.67)\end{array}$ & & & & 1980 & $-4.66^{*}$ & 0.60 & 0.80 \\
\hline RS: $C A G D P=f(C, B D G D P)$ & $\begin{array}{l}-0.76^{*} \\
(-2.00) \\
\end{array}$ & $\begin{array}{l}2.22^{* *} \\
(4.05)\end{array}$ & $\begin{array}{c}0.25 \\
(1.56)\end{array}$ & & $\begin{array}{l}-0.16 \\
(-0.85) \\
\end{array}$ & & 1980 & -4.82 & 0.60 & 0.84 \\
\hline \multicolumn{11}{|c|}{ Canada:1960-2000: Two regressors } \\
\hline SC: $C A G D P=f(C, B D G D P, r)$ & $\begin{array}{c}-0.85 \\
(-2.00)\end{array}$ & & $\begin{array}{c}-0.01 \\
(-0.07)\end{array}$ & $\begin{array}{l}0.27^{*} \\
(2.66)\end{array}$ & & & & -2.84 & 0.17 & 0.49 \\
\hline LS: CAGDP=f(C, BDGDP, r $)$ & $\begin{array}{c}-0.97 \\
(-3.20)\end{array}$ & $\begin{array}{l}2.60^{* *} \\
(6.23)\end{array}$ & $\begin{array}{c}0.13 \\
(1.63)\end{array}$ & $\begin{array}{c}-0.01 \\
(-0.10)\end{array}$ & & & 1980 & $-4.61^{*}$ & 0.60 & 0.81 \\
\hline RS: $C A G D P=f(C, B D G D P, r)$ & $\begin{array}{c}-0.71 \\
(-1.45)\end{array}$ & $\begin{array}{l}2.24^{* *} \\
(2.98)\end{array}$ & $\begin{array}{c}0.26 \\
(1.52)\end{array}$ & $\begin{array}{c}-0.03 \\
(-0.18)\end{array}$ & $\begin{array}{c}-0.17 \\
(-0.86)\end{array}$ & $\begin{array}{c}0.01 \\
(0.03)\end{array}$ & 1980 & -4.70 & 0.60 & 0.85 \\
\hline \multicolumn{11}{|c|}{ Denmark:1960-1999: One regressor } \\
\hline SC: $\mathrm{CAGDP}=\mathrm{f}(\mathrm{C}, \mathrm{BDGDP})$ & $\begin{array}{l}-1.64^{\star \star} \\
(-5.08)\end{array}$ & & $\begin{array}{c}-0.04 \\
(-0.36)\end{array}$ & & & & & -2.17 & 0.003 & 0.54 \\
\hline LS: CAGDP=f(C, BDGDP $)$ & $\begin{array}{r}-2.63^{* *} \\
(-10.33)\end{array}$ & $\begin{array}{c}3.30 \\
(7.09)\end{array}$ & $\begin{array}{l}-0.002 \\
(-0.02)\end{array}$ & & & & 1988 & $-5.25^{\star \star}$ & 0.58 & 1.32 \\
\hline RS: $C A G D P=f(C, B D G D P)$ & $\begin{array}{l}-2.38^{* \star} \\
(-8.58) \\
\end{array}$ & $\begin{array}{l}2.18^{* *} \\
(4.83) \\
\end{array}$ & $\begin{array}{c}0.16 \\
(1.64) \\
\end{array}$ & & $\begin{array}{l}-0.94^{* *} \\
(-4.93) \\
\end{array}$ & & 1985 & $-5.71^{\star \star}$ & 0.57 & 1.66 \\
\hline \multicolumn{11}{|c|}{ Denmark:1960-1999: Two regressors } \\
\hline SC: CAGDP=f(C, BDGDP, r $)$ & $\begin{array}{l}-2.05^{* *} \\
(-6.02)\end{array}$ & & $\begin{array}{c}-0.06 \\
(-0.54)\end{array}$ & $\begin{array}{l}0.29^{*} \\
(2.57)\end{array}$ & & & & -2.97 & 0.15 & 0.86 \\
\hline LS: CAGDP=f(C, BDGDP, r $)$ & $\begin{array}{l}-2.64^{\star \star} \\
(-10.03)\end{array}$ & $\begin{array}{l}3.26^{* *} \\
(6.01)\end{array}$ & $\begin{array}{l}-0.003 \\
(-0.04)\end{array}$ & $\begin{array}{c}0.02 \\
(0.17)\end{array}$ & & & 1988 & $-5.26^{\star *}$ & 0.57 & 1.33 \\
\hline RS: CAGDP $=f(C, B D G D P, r)$ & $\begin{array}{l}-2.38^{* *} \\
(-8.27)\end{array}$ & $\begin{array}{l}2.34^{* *} \\
(2.99)\end{array}$ & $\begin{array}{c}0.16 \\
(1.59)\end{array}$ & $\begin{array}{c}-0.01 \\
(-0.08)\end{array}$ & $\begin{array}{l}-0.96^{* *} \\
(-4.58)\end{array}$ & $\begin{array}{c}-0.04 \\
(-0.17)\end{array}$ & 1985 & $-5.94^{* \star}$ & 0.57 & 1.68 \\
\hline \multicolumn{11}{|c|}{ Finland:1960-1998: One regressor } \\
\hline SC: $\mathrm{CAGDP}=\mathrm{f}(\mathrm{C}, \mathrm{BDGDP})$ & $\begin{array}{l}-1.46^{\star \star} \\
(-2.79)\end{array}$ & & $\begin{array}{c}-0.09 \\
(-0.79)\end{array}$ & & & & & -2.50 & 0.01 & 0.44 \\
\hline LS: CAGDP=f(C, BDGDP $)$ & $\begin{array}{l}-1.62^{* *} \\
(-4.14)\end{array}$ & $\begin{array}{l}7.21^{* *} \\
(5.58)\end{array}$ & $\begin{array}{l}0.43^{* *} \\
(3.34)\end{array}$ & & & & 1992 & -4.00 & 0.47 & 0.79 \\
\hline RS: $C A G D P=f(C, B D G D P)$ & $\begin{array}{l}-2.06^{* \star} \\
(-4.48) \\
\end{array}$ & $\begin{array}{l}7.85^{* *} \\
(4.68)\end{array}$ & $\begin{array}{r}-0.27 \\
(-0.91) \\
\end{array}$ & & $\begin{array}{l}0.85^{*} \\
(2.48)\end{array}$ & & 1991 & -4.27 & 0.40 & 0.94 \\
\hline \multicolumn{11}{|c|}{ Finland:1960-1998: Two regressors } \\
\hline SC: $C A G D P=f(C, B D G D P, r)$ & $\begin{array}{l}-1.57^{* *} \\
(-3.04)\end{array}$ & & $\begin{array}{c}-0.02 \\
(-0.16)\end{array}$ & $\begin{array}{c}0.23 \\
(1.66)\end{array}$ & & & & -3.45 & 0.09 & 0.43 \\
\hline LS: CAGDP $=f(C, B D G D P, r)$ & $\begin{array}{l}-1.68^{* *} \\
(-4.31)\end{array}$ & $\begin{array}{l}6.93^{* *} \\
(5.36)\end{array}$ & $\begin{array}{l}0.46^{* *} \\
(3.53)\end{array}$ & $\begin{array}{c}0.14 \\
(1.34)\end{array}$ & & & 1992 & -3.87 & 0.50 & 0.78 \\
\hline RS: CAGDP $=f(C, B D G D P, r)$ & $\begin{array}{l}-2.05^{* *} \\
(-4.02)\end{array}$ & $\begin{array}{l}9.36^{* *} \\
(3.46)\end{array}$ & $\begin{array}{c}-0.27 \\
(-0.85)\end{array}$ & $\begin{array}{c}0.24 \\
(1.85)\end{array}$ & $\begin{array}{l}0.04 \\
(0.09)\end{array}$ & $\begin{array}{l}-2.54^{*} \\
(-2.51)\end{array}$ & 1990 & -4.33 & 0.34 & 0.95 \\
\hline
\end{tabular}


Table B2 continued

\begin{tabular}{|c|c|c|c|c|c|c|c|c|c|c|}
\hline $\begin{array}{l}\text { Country, sample periods } \\
\text { And model types }\end{array}$ & $\mu_{1}$ & $\mu_{1} \varphi_{1 \tau}$ & $\beta_{1}$ & $\beta_{2}$ & $\beta_{3} \varphi_{1 \tau}$ & $\beta_{4} \varphi_{1 \tau}$ & $\begin{array}{c}\text { Break } \\
\text { year }\end{array}$ & ADF & $\mathrm{R}^{2}$ & DW \\
\hline \multicolumn{11}{|c|}{ France:1960-1997: One regressor } \\
\hline SC: $\mathrm{CAGDP}=\mathrm{f}(\mathrm{C}, \mathrm{BDGDP})$ & $\begin{array}{c}-0.33 \\
(-1.65)\end{array}$ & & $\begin{array}{c}-0.16 \\
(-2.01)\end{array}$ & & & & & -3.28 & 0.10 & 1.12 \\
\hline LS: CAGDP=f(C, BDGDP) & $\begin{array}{c}-0.21 \\
(-1.06) \\
\end{array}$ & $\begin{array}{l}1.14^{*} \\
(2.50)\end{array}$ & $\begin{array}{c}0.02 \\
(0.20)\end{array}$ & & & & 1991 & $-4.66^{*}$ & 0.23 & 1.47 \\
\hline RS: $C A G D P=f(C, B D G D P)$ & $\begin{array}{c}-0.07 \\
(-0.30)\end{array}$ & $\begin{array}{c}-0.76 \\
(-1.41) \\
\end{array}$ & $\begin{array}{c}0.14 \\
(0.95) \\
\end{array}$ & & $\begin{array}{l}-0.59^{* \star} \\
(-4.66)\end{array}$ & & 1984 & -4.79 & 0.24 & 1.61 \\
\hline \multicolumn{11}{|c|}{ France:1960-1997: Two regressors } \\
\hline SC: CAGDP $=f(C, B D G D P, r)$ & $\begin{array}{c}-0.10 \\
(-0.42) \\
\end{array}$ & & $\begin{array}{c}-0.08 \\
(-0.86)\end{array}$ & $\begin{array}{c}0.10 \\
(1.67)\end{array}$ & & & & -3.20 & 0.17 & 1.08 \\
\hline LS: CAGDP $=f(C, B D G D P, r)$ & $\begin{array}{c}-0.08 \\
(-0.32)\end{array}$ & $\begin{array}{l}0.99^{*} \\
(2.08)\end{array}$ & $\begin{array}{l}0.05 \\
(0.45)\end{array}$ & $\begin{array}{l}0.06 \\
(1.06)\end{array}$ & & & 1991 & $-5.17^{*}$ & 0.26 & 1.40 \\
\hline RS: CAGDP $=f(C, B D G D P, r)$ & $\begin{array}{c}0.03 \\
(0.11)\end{array}$ & $\begin{array}{c}-0.19 \\
(-0.14)\end{array}$ & $\begin{array}{c}0.12 \\
(0.95)\end{array}$ & $\begin{array}{c}0.07 \\
(1.09)\end{array}$ & $\begin{array}{c}-0.16 \\
(-0.51)\end{array}$ & $\begin{array}{c}0.32 \\
(0.37)\end{array}$ & 1991 & -5.25 & 0.29 & 1.52 \\
\hline \multicolumn{11}{|c|}{ Germany:1960-1998: One regressor } \\
\hline SC: $C A G D P=f(C, B D G D P)$ & $\begin{array}{l}1.16^{* *} \\
(2.96)\end{array}$ & & $\begin{array}{l}0.21 \\
(0.87)\end{array}$ & & & & & -2.92 & 0.02 & 0.50 \\
\hline LS: CAGDP=f(C, BDGDP $)$ & $\begin{array}{c}0.57 \\
(0.92)\end{array}$ & $\begin{array}{c}0.89 \\
(1.26)\end{array}$ & $\begin{array}{l}0.31 \\
(1.21)\end{array}$ & & & & 1968 & -3.06 & 0.06 & 0.54 \\
\hline RS: CAGDP=f(C, BDGDP) & $\begin{array}{l}0.78^{*} \\
(2.06)\end{array}$ & $\begin{array}{l}3.89^{*} \\
(3.66) \\
\end{array}$ & $\begin{array}{c}-0.12 \\
(-0.42) \\
\end{array}$ & & $\begin{array}{l}2.31^{* *} \\
(3.69)\end{array}$ & & 1977 & -3.37 & 0.31 & 1.00 \\
\hline \multicolumn{11}{|c|}{ Germany:1960-1998: Two regressors } \\
\hline SC: $C A G D P=f(C, B D G D P, r)$ & $\begin{array}{l}0.97^{*} \\
(2.18)\end{array}$ & & $\begin{array}{c}0.21 \\
(0.86)\end{array}$ & $\begin{array}{l}0.14 \\
(0.86)\end{array}$ & & & & -2.42 & 0.04 & 0.57 \\
\hline LS: CAGDP=f(C, BDGDP, r) & $\begin{array}{l}1.08^{*} \\
(2.61)\end{array}$ & $\begin{array}{c}-1.83 \\
(-2.68)\end{array}$ & $\begin{array}{c}0.05 \\
(0.23)\end{array}$ & $\begin{array}{l}0.17 \\
(1.13)\end{array}$ & & & 1992 & -3.19 & 0.20 & 0.77 \\
\hline RS: CAGDP $=f(C, B D G D P, r)$ & $\begin{array}{c}0.78 \\
(1.87)\end{array}$ & $\begin{array}{l}3.32^{*} \\
(2.56)\end{array}$ & $\begin{array}{c}0.09 \\
(0.37)\end{array}$ & $\begin{array}{c}-0.12 \\
(-0.56)\end{array}$ & $\begin{array}{l}2.20^{* *} \\
(3.20)\end{array}$ & $\begin{array}{c}0.44 \\
(1.22)\end{array}$ & 1984 & -3.90 & 0.33 & 1.18 \\
\hline \multicolumn{11}{|c|}{ Greece:1960-1999: One regressor } \\
\hline SC: $\mathrm{CAGDP}=\mathrm{f}(\mathrm{C}, \mathrm{BDGDP})$ & $\begin{array}{l}-3.70^{* *} \\
(-7.58)\end{array}$ & & $\begin{array}{l}0.07 \\
(1.14)\end{array}$ & & & & & -3.61 & 0.03 & 0.95 \\
\hline LS: CAGDP=f(C, BDGDP $)$ & $\begin{array}{l}-3.46^{* *} \\
(-7.72)\end{array}$ & $\begin{array}{l}2.41^{* *} \\
(3.05)\end{array}$ & $\begin{array}{c}0.24 \\
(3.01)\end{array}$ & & & & 1987 & $-4.92^{*}$ & 0.23 & 1.26 \\
\hline RS: $C A G D P=f(C, B D G D P)$ & $\begin{array}{l}-2.43^{* \star} \\
(-5.77)\end{array}$ & $\begin{array}{l}-2.16 \\
(-1.85) \\
\end{array}$ & $\begin{array}{l}0.50^{* \star} \\
(5.91)\end{array}$ & & $\begin{array}{l}0.50^{* \star} \\
(5.91)\end{array}$ & & 1987 & $-6.49^{* \star}$ & 0.52 & 1.54 \\
\hline \multicolumn{11}{|c|}{ Greece:1960-1999: Two regressors } \\
\hline SC: $C A G D P=f(C, B D G D P, r)$ & $\begin{array}{l}-3.58^{* *} \\
(-9.58)\end{array}$ & & $\begin{array}{c}0.09 \\
(1.88)\end{array}$ & $\begin{array}{l}0.20^{* *} \\
(4.94)\end{array}$ & & & & $-4.91^{\star \star}$ & 0.41 & 1.53 \\
\hline LS: CAGDP $=f(C, B D G D P, r)$ & $\begin{array}{l}-3.67^{* *} \\
(-9.13)\end{array}$ & $\begin{array}{c}-1.25 \\
(-1.52)\end{array}$ & $\begin{array}{c}-0.01 \\
(-0.14)\end{array}$ & $\begin{array}{l}0.21^{* *} \\
(5.25)\end{array}$ & & & 1982 & $-5.60^{\star \star}$ & 0.45 & 1.74 \\
\hline RS: CAGDP $=f(C, B D G D P, r)$ & $\begin{array}{l}-2.70^{* *} \\
(-6.64)\end{array}$ & $\begin{array}{l}-2.55^{*} \\
(-2.11)\end{array}$ & $\begin{array}{l}0.38^{* *} \\
(4.28)\end{array}$ & $\begin{array}{l}0.12^{\star} \\
(2.41)\end{array}$ & $\begin{array}{l}-0.47^{* *} \\
(-3.78)\end{array}$ & $\begin{array}{l}0.05 \\
(0.36)\end{array}$ & 1987 & $-5.97^{\star \star}$ & 0.60 & 1.76 \\
\hline
\end{tabular}


Table B2 continued

\begin{tabular}{|c|c|c|c|c|c|c|c|c|c|c|}
\hline $\begin{array}{l}\text { Country, sample periods } \\
\text { And model types }\end{array}$ & $\mu_{1}$ & $\mu_{1} \varphi_{1 \tau}$ & $\beta_{1}$ & $\beta_{2}$ & $\beta_{3} \varphi_{1 \tau}$ & $\beta_{4} \varphi_{1 \tau}$ & $\begin{array}{c}\text { Break } \\
\text { year }\end{array}$ & ADF & $\mathrm{R}^{2}$ & DW \\
\hline \multicolumn{11}{|c|}{ Iceland:1960-1997: One regressor } \\
\hline SC: $\mathrm{CAGDP}=\mathrm{f}(\mathrm{C}, \mathrm{BDGDP})$ & $\begin{array}{c}-0.44 \\
(-0.39) \\
\end{array}$ & & $\begin{array}{l}0.76^{*} \\
(2.15) \\
\end{array}$ & & & & & -1.2 & 0.11 & 1.55 \\
\hline LS: CAGDP=f(C, BDGDP) & $\begin{array}{c}-0.88 \\
(-0.77) \\
\end{array}$ & $\begin{array}{c}1.72 \\
(1.46) \\
\end{array}$ & $\begin{array}{l}0.85^{*} \\
(2.40)\end{array}$ & & & & 1983 & $-6.11^{* *}$ & 0.16 & 1.61 \\
\hline RS: CAGDP=f(C, BDGDP) & $\begin{array}{c}0.55 \\
(0.46) \\
\end{array}$ & $\begin{array}{c}-3.13 \\
(-1.31)\end{array}$ & $\begin{array}{l}1.42^{\star *} \\
(3.53)\end{array}$ & & $\begin{array}{l}-1.72^{*} \\
(-2.41)\end{array}$ & & 1985 & $-6.36^{* *}$ & 0.29 & 1.68 \\
\hline \multicolumn{11}{|c|}{ Iceland:1960-1997: Two regressors } \\
\hline SC: $C A G D P=f(C, B D G D P, r)$ & $\begin{array}{c}-0.29 \\
(-0.25)\end{array}$ & & $\begin{array}{c}0.71 \\
(2.01)\end{array}$ & $\begin{array}{c}0.03 \\
(1.03)\end{array}$ & & & & $-5.99^{* *}$ & 0.14 & 1.71 \\
\hline LS: CAGDP=f(C, BDGDP, r) & $\begin{array}{c}-0.75 \\
(-0.62) \\
\end{array}$ & $\begin{array}{c}1.46 \\
(1.09)\end{array}$ & $\begin{array}{l}0.82^{*} \\
(2.22)\end{array}$ & $\begin{array}{c}0.02 \\
(0.41)\end{array}$ & & & 1983 & $-6.15^{\star *}$ & 0.17 & 1.66 \\
\hline RS: $C A G D P=f(C, B D G D P, r)$ & $\begin{array}{c}0.58 \\
(0.46) \\
\end{array}$ & $\begin{array}{c}-1.89 \\
(-0.68)\end{array}$ & $\begin{array}{l}1.37^{\star *} \\
(3.25)\end{array}$ & $\begin{array}{c}0.01 \\
(0.12) \\
\end{array}$ & & & 1986 & $-6.58^{\star *}$ & 0.32 & 1.77 \\
\hline \multicolumn{11}{|c|}{ |reland:1960-1999: One regressor } \\
\hline SC: $C A G D P=f(C, B D G D P)$ & $\begin{array}{c}-0.38 \\
(-0.10) \\
\end{array}$ & & $\begin{array}{l}2.12^{* *} \\
(4.73)\end{array}$ & & & & & -3.40 & 0.37 & 0.92 \\
\hline LS: CAGDP=f(C, BDGDP) & $\begin{array}{c}-14.56^{\star *} \\
(-3.20)\end{array}$ & $\begin{array}{c}18.87^{\star *} \\
(4.17)\end{array}$ & $\begin{array}{l}1.09^{*} \\
(2.41)\end{array}$ & & & & 1984 & $-5.36^{* *}$ & 0.57 & 1.44 \\
\hline RS: CAGDP=f(C, BDGDP) & $\begin{array}{c}-8.16 \\
(-1.39) \\
\end{array}$ & $\begin{array}{l}11.82 \\
(1.77) \\
\end{array}$ & $\begin{array}{l}2.03^{\star *} \\
(3.10) \\
\end{array}$ & & $\begin{array}{c}-0.99 \\
(-1.23) \\
\end{array}$ & & 1982 & $-5.48^{* *}$ & 0.63 & 1.53 \\
\hline \multicolumn{11}{|c|}{ Ireland:1960-1999: Two regressors } \\
\hline SC: $C A G D P=f(C, B D G D P, r)$ & $\begin{array}{c}-6.15 \\
(-1.42) \\
\end{array}$ & & $\begin{array}{l}1.59^{* *} \\
(3.24)\end{array}$ & $\begin{array}{l}1.19^{*} \\
(2.21)\end{array}$ & & & & -4.02 & 0.44 & 1.19 \\
\hline LS: CAGDP=f(C, BDGDP, r $)$ & $\begin{array}{l}-12.94^{*} \\
(-2.51)\end{array}$ & $\begin{array}{l}10.20^{*} \\
(2.19)\end{array}$ & $\begin{array}{l}1.71^{* *} \\
(3.63)\end{array}$ & $\begin{array}{l}1.14^{*} \\
(2.22)\end{array}$ & & & 1970 & $-5.36^{* *}$ & 0.50 & 1.33 \\
\hline RS: CAGDP $=f(C, B D G D P, r)$ & $\begin{array}{l}-16.40 \\
(-1.68) \\
\end{array}$ & $\begin{array}{l}13.19 \\
(1.15) \\
\end{array}$ & $\begin{array}{c}0.68 \\
(0.52)\end{array}$ & $\begin{array}{c}0.34 \\
(0.38)\end{array}$ & $\begin{array}{c}1.02 \\
(0.72) \\
\end{array}$ & $\begin{array}{c}1.07 \\
(0.87) \\
\end{array}$ & 1979 & $-5.67^{\star \star}$ & 0.53 & 1.42 \\
\hline \multicolumn{11}{|c|}{ |taly:1960-1998: One regressor } \\
\hline SC: $C A G D P=f(C, B D G D P)$ & $\begin{array}{l}2.19^{\star *} \\
(3.63) \\
\end{array}$ & & $\begin{array}{l}0.23^{\star *} \\
(3.35)\end{array}$ & & & & & -3.84 & 0.23 & 1.12 \\
\hline LS: CAGDP=f(C, BDGDP) & $\begin{array}{l}2.18^{\star \star} \\
(3.62) \\
\end{array}$ & $\begin{array}{c}-0.96 \\
(-1.12) \\
\end{array}$ & $\begin{array}{c}0.14 \\
(1.43) \\
\end{array}$ & & & & 1973 & -4.44 & 0.26 & 1.02 \\
\hline RS: CAGDP=f(C, BDGDP) & $\begin{array}{c}-1.36 \\
(-0.69) \\
\end{array}$ & $\begin{array}{l}4.44^{*} \\
(2.09)\end{array}$ & $\begin{array}{c}-1.01 \\
(-1.24) \\
\end{array}$ & & $\begin{array}{c}1.32 \\
(1.61) \\
\end{array}$ & & 1966 & -4.50 & 0.32 & 1.37 \\
\hline \multicolumn{11}{|c|}{ |taly:1960-1998: Two regressors } \\
\hline SC: $C A G D P=f(C, B D G D P, r)$ & $\begin{array}{l}2.26^{\star *} \\
(3.78)\end{array}$ & & $\begin{array}{l}0.25^{\star *} \\
(3.60)\end{array}$ & $\begin{array}{c}0.09 \\
(1.38)\end{array}$ & & & & -3.99 & 0.27 & 1.17 \\
\hline LS: CAGDP=f(C, BDGDP, r $)$ & $\begin{array}{l}1.71^{*} \\
(2.40)\end{array}$ & $\begin{array}{c}1.27 \\
(1.37)\end{array}$ & $\begin{array}{l}0.31^{* *} \\
(3.77)\end{array}$ & $\begin{array}{c}0.08 \\
(1.30) \\
\end{array}$ & & & 1965 & -4.37 & 0.31 & 1.36 \\
\hline RS: $C A G D P=f(C, B D G D P, r)$ & $\begin{array}{c}1.15 \\
(1.19) \\
\end{array}$ & $\begin{array}{c}0.89 \\
(0.60) \\
\end{array}$ & $\begin{array}{c}-0.14 \\
(-0.61) \\
\end{array}$ & $\begin{array}{l}0.59^{*} \\
(2.44)\end{array}$ & $\begin{array}{c}0.37 \\
(1.49) \\
\end{array}$ & $\begin{array}{l}-0.52^{*} \\
(-2.09) \\
\end{array}$ & 1974 & -4.78 & 0.38 & 1.16 \\
\hline
\end{tabular}


Table B2 continued

\begin{tabular}{|c|c|c|c|c|c|c|c|c|c|c|}
\hline $\begin{array}{l}\text { Country, sample periods } \\
\text { and model types }\end{array}$ & $\mu_{1}$ & $\mu_{1} \varphi_{1 \tau}$ & $\beta_{1}$ & $\beta_{2}$ & $\beta_{3} \varphi_{1 \tau}$ & $\beta_{4} \varphi_{1 \tau}$ & $\begin{array}{l}\text { Break } \\
\text { year }\end{array}$ & ADF & $\mathrm{R}^{2}$ & DW \\
\hline \multicolumn{11}{|c|}{ Japan: 1970 - 1993, one regressor } \\
\hline SC: $C A G D P=f(C, B D G D P)$ & $\begin{array}{l}2.10^{\star \star} \\
(4.12)\end{array}$ & & $\begin{array}{c}0.14 \\
(1.30)\end{array}$ & & & & & -2.71 & 0.07 & 0.59 \\
\hline LS: CAGDP=f(C, BDGDP) & $\begin{array}{c}0.90 \\
(1.82) \\
\end{array}$ & $\begin{array}{c}1.89 \\
(3.99) \\
\end{array}$ & $\begin{array}{c}0.08 \\
(0.87) \\
\end{array}$ & & & & 1982 & $-5.79^{*}$ & 0.47 & 1.09 \\
\hline RS: CAGDP=f(C, BDGDP) & $\begin{array}{c}1.09 \\
(1.80)\end{array}$ & $\begin{array}{c}1.52 \\
(1.88)\end{array}$ & $\begin{array}{c}0.12 \\
(1.01)\end{array}$ & & $\begin{array}{l}-0.10 \\
(-0.56)\end{array}$ & & 1982 & $-6.22^{*}$ & 0.48 & 1.16 \\
\hline \multicolumn{11}{|c|}{ Japan: 1970 - 1993, two regressors } \\
\hline SC: $C A G D P=f(C, B D G D P, r)$ & $\begin{array}{l}2.25^{\star \star} \\
(5.28)\end{array}$ & & $\begin{array}{c}0.17 \\
(1.87)\end{array}$ & $\begin{array}{l}0.22^{* *} \\
(3.28) \\
\end{array}$ & & & & -3.66 & 0.38 & 1.04 \\
\hline LS: CAGDP=f(C, BDGDP, r $)$ & $\begin{array}{l}1.28^{*} \\
(2.17)\end{array}$ & $\begin{array}{c}1.39^{*} \\
(2.19)\end{array}$ & $\begin{array}{c}0.11 \\
(1.18)\end{array}$ & $\begin{array}{c}0.10 \\
(1.17)\end{array}$ & & & 1982 & $-4.90^{*}$ & 0.51 & 1.11 \\
\hline RS: $C A G D P=f(C, B D G D P, r)$ & $\begin{array}{l}1.56^{*} \\
(2.10) \\
\end{array}$ & $\begin{array}{c}0.16 \\
(0.15) \\
\end{array}$ & $\begin{array}{c}0.11 \\
(0.79) \\
\end{array}$ & $\begin{array}{c}0.13 \\
(1.37) \\
\end{array}$ & $\begin{array}{c}0.11 \\
(0.61)\end{array}$ & $\begin{array}{c}0.53 \\
(1.66) \\
\end{array}$ & 1980 & -4.70 & 0.49 & 1.21 \\
\hline \multicolumn{11}{|c|}{ Luxembourg: 1973 - 1997: One regressor } \\
\hline SC: $C A G D P=f(C, B D G D P)$ & $\begin{array}{l}7.79^{* \star} \\
(6.11)\end{array}$ & & $\begin{array}{c}0.53 \\
(1.68)\end{array}$ & & & & & -2.51 & 0.11 & 0.77 \\
\hline LS: CAGDP=f(C, BDGDP) & $\begin{array}{l}6.10^{* *} \\
(3.89)\end{array}$ & $\begin{array}{c}4.45 \\
(1.74)\end{array}$ & $\begin{array}{l}0.71^{*} \\
(2.22)\end{array}$ & & & & 1990 & -3.31 & 0.22 & 1.00 \\
\hline RS: CAGDP=f(C, BDGDP) & $\begin{array}{l}5.57^{*} \\
(2.59) \\
\end{array}$ & $\begin{array}{c}2.13 \\
(0.83) \\
\end{array}$ & $\begin{array}{l}2.94^{* *} \\
(3.09)\end{array}$ & & $\begin{array}{l}-2.63^{*} \\
(-2.64) \\
\end{array}$ & & 1982 & -3.51 & 0.34 & 1.35 \\
\hline \multicolumn{11}{|c|}{ Luxembourg: 1973 - 1997: Two regressors } \\
\hline SC: $C A G D P=f(C, B D G D P, r)$ & $\begin{array}{l}9.18^{\star *} \\
(5.59)\end{array}$ & & $\begin{array}{c}0.61 \\
(1.92)\end{array}$ & $\begin{array}{l}-0.52 \\
(-1.31)\end{array}$ & & & & -2.53 & 0.17 & 0.84 \\
\hline LS: CAGDP=f(C, BDGDP, $r)$ & $\begin{array}{l}7.65^{\star *} \\
(4.95)\end{array}$ & $\begin{array}{l}6.94^{*} \\
(2.76)\end{array}$ & $\begin{array}{l}0.95^{\star \star} \\
(3.12)\end{array}$ & $\begin{array}{l}-0.94^{*} \\
(-2.48)\end{array}$ & & & 1990 & -4.35 & 0.39 & 1.53 \\
\hline RS: $C A G D P=f(C, B D G D P, r)$ & $\begin{array}{l}5.02^{*} \\
(2.62)\end{array}$ & $\begin{array}{c}6.30 \\
(1.44)\end{array}$ & $\begin{array}{l}2.97^{* *} \\
(3.35)\end{array}$ & $\begin{array}{l}-1.40 \\
(-1.97)\end{array}$ & $\begin{array}{l}-2.60^{*} \\
(-2.78)\end{array}$ & $\begin{array}{c}0.71 \\
(0.66)\end{array}$ & 1983 & -5.19 & 0.48 & 1.55 \\
\hline \multicolumn{11}{|c|}{ Netherlands:1960-1998: One regressor } \\
\hline SC: $\mathrm{CAGDP}=\mathrm{f}(\mathrm{C}, \mathrm{BDGDP})$ & $\begin{array}{l}1.98^{\star \star} \\
(3.64)\end{array}$ & & $\begin{array}{c}-0.08 \\
(-0.51)\end{array}$ & & & & & -2.21 & 0.01 & 0.46 \\
\hline LS: CAGDP=f(C, BDGDP) & $\begin{array}{l}1.24^{*} \\
(2.48)\end{array}$ & $\begin{array}{l}2.83^{\star \star} \\
(3.88)\end{array}$ & $\begin{array}{c}0.31 \\
(1.77) \\
\end{array}$ & & & & 1975 & $-4.75^{\star}$ & 0.30 & 0.87 \\
\hline RS: CAGDP=f(C, BDGDP) & $\begin{array}{l}2.24^{* *} \\
(3.54)\end{array}$ & $\begin{array}{c}1.27 \\
(1.34)\end{array}$ & $\begin{array}{l}1.26^{* *} \\
(2.90)\end{array}$ & & $\begin{array}{l}-1.11^{*} \\
(-2.36)\end{array}$ & & 1975 & -4.55 & 0.40 & 0.88 \\
\hline \multicolumn{11}{|c|}{ Netherlands:1960-1998: Two regressors } \\
\hline SC: $C A G D P=f(C, B D G D P, r)$ & $\begin{array}{l}1.96^{* *} \\
(3.56)\end{array}$ & & $\begin{array}{c}-0.06 \\
(-0.34)\end{array}$ & $\begin{array}{c}0.07 \\
(0.53) \\
\end{array}$ & & & & -2.26 & 0.01 & 0.49 \\
\hline LS: $C A G D P=f(C, B D G D P, r)$ & $\begin{array}{l}1.30^{\star *} \\
(3.51)\end{array}$ & $\begin{array}{l}4.16^{* *} \\
(7.01)\end{array}$ & $\begin{array}{c}0.25^{\star} \\
(2.09)\end{array}$ & $\begin{array}{c}-0.32 \\
(-3.08)\end{array}$ & & & 1981 & $-4.65^{\star}$ & 0.59 & 1.22 \\
\hline RS: $C A G D P=f(C, B D G D P, r)$ & $\begin{array}{l}1.64^{\star *} \\
(3.32)\end{array}$ & $\begin{array}{l}3.73^{\star *} \\
(3.77)\end{array}$ & $\begin{array}{c}0.45^{*} \\
(2.01) \\
\end{array}$ & $\begin{array}{c}-0.28^{*} \\
(-2.23) \\
\end{array}$ & $\begin{array}{c}-0.27 \\
(-1.01)\end{array}$ & $\begin{array}{c}-0.11 \\
(-0.47) \\
\end{array}$ & 1981 & -4.72 & 0.60 & 1.18 \\
\hline
\end{tabular}


Table B2 continued

\begin{tabular}{|c|c|c|c|c|c|c|c|c|c|c|}
\hline $\begin{array}{c}\text { Country, sample periods } \\
\text { and model types }\end{array}$ & $\mu_{1}$ & $\mu_{1} \varphi_{1 \tau}$ & $\beta_{1}$ & $\beta_{2}$ & $\beta_{3} \varphi_{1 \tau}$ & $\beta_{4} \varphi_{1 \tau}$ & $\begin{array}{c}\text { Break } \\
\text { year }\end{array}$ & ADF & $\mathrm{R}^{2}$ & DW \\
\hline \multicolumn{11}{|c|}{ New Zealand:1960-2000: One regressor } \\
\hline SC: $C A G D P=f(C, B D G D P)$ & $\begin{array}{c}-3.38 \\
(-6.00) \\
\end{array}$ & & $\begin{array}{c}0.12 \\
(0.96) \\
\end{array}$ & & & & & -3.33 & 0.02 & 0.88 \\
\hline LS: CAGDP=f(C, BDGDP) & $\begin{array}{c}-0.54 \\
(-0.73)\end{array}$ & $\begin{array}{l}-3.96^{* *} \\
(-4.83)\end{array}$ & $\begin{array}{c}0.14 \\
(1.35)\end{array}$ & & & & 1972 & $-5.59^{* *}$ & 0.39 & 1.55 \\
\hline RS: CAGDP=f(C, BDGDP) & $\begin{array}{c}1.70 \\
(1.50)\end{array}$ & $\begin{array}{l}-6.34^{\star *} \\
(-5.09)\end{array}$ & $\begin{array}{l}1.14^{\star *} \\
(3.89)\end{array}$ & & $\begin{array}{c}-1.07^{* *} \\
(-3.43)\end{array}$ & & 1977 & $-6.30^{\star *}$ & 0.43 & 1.99 \\
\hline \multicolumn{11}{|c|}{ New Zealand:1960-2000: Two regressors } \\
\hline SC: $C A G D P=f(C, B D G D P, r)$ & $\begin{array}{c}-3.47^{\star *} \\
(4.27)\end{array}$ & & $\begin{array}{c}0.10 \\
(0.64)\end{array}$ & $\begin{array}{c}0.02 \\
(0.17)\end{array}$ & & & & -3.31 & 0.02 & 0.87 \\
\hline LS: CAGDP=f(C, BDGDP, $r)$ & $\begin{array}{c}-1.08 \\
(-1.49)\end{array}$ & $\begin{array}{c}-6.26^{* *} \\
(-5.84)\end{array}$ & $\begin{array}{l}0.37^{* *} \\
(2.97)\end{array}$ & $\begin{array}{l}0.36^{\star *} \\
(3.26)\end{array}$ & & & 1984 & $-5.78^{\star *}$ & 0.49 & 1.73 \\
\hline RS: CAGDP=f(C, BDGDP, $r)$ & $\begin{array}{c}0.50 \\
(0.37)\end{array}$ & $\begin{array}{l}-5.10^{* *} \\
(-3.30)\end{array}$ & $\begin{array}{c}0.82^{*} \\
(2.46) \\
\end{array}$ & $\begin{array}{c}0.28 \\
(1.58)\end{array}$ & $\begin{array}{l}-0.72^{*} \\
(-2.03) \\
\end{array}$ & $\begin{array}{c}-0.30 \\
-(1.41) \\
\end{array}$ & 1976 & $-6.05^{* *}$ & 0.49 & 1.90 \\
\hline \multicolumn{11}{|c|}{ Norway:1960-1998: One regressor } \\
\hline SC: $C A G D P=f(C, B D G D P)$ & $\begin{array}{c}0.13 \\
(0.18) \\
\end{array}$ & & $\begin{array}{l}0.69^{*} \\
(2.67)\end{array}$ & & & & & -2.62 & 0.16 & 0.62 \\
\hline LS: CAGDP=f(C, BDGDP) & $\begin{array}{c}-1.13 \\
(-1.57) \\
\end{array}$ & $\begin{array}{l}4.54^{* *} \\
(3.68)\end{array}$ & $\begin{array}{l}0.71^{* *} \\
(3.16)\end{array}$ & & & & 1988 & -4.54 & 0.39 & 0.88 \\
\hline RS: CAGDP=f(C, BDGDP) & $\begin{array}{c}0.25 \\
(0.30)\end{array}$ & $\begin{array}{c}1.00 \\
(0.81)\end{array}$ & $\begin{array}{l}1.33^{\star \star} \\
(4.42)\end{array}$ & & $\begin{array}{c}-1.59^{\star *} \\
(-3.64)\end{array}$ & & 1985 & $-5.31^{*}$ & 0.46 & 0.97 \\
\hline \multicolumn{11}{|c|}{ Norway:1960-1998: Two regressors } \\
\hline SC: $C A G D P=f(C, B D G D P, r)$ & $\begin{array}{c}-0.52 \\
(-0.72)\end{array}$ & & $\begin{array}{c}0.59^{*} \\
(2.43)\end{array}$ & $\begin{array}{l}0.39^{*} \\
(2.63)\end{array}$ & & & & -3.10 & 0.30 & 0.82 \\
\hline LS: CAGDP=f(C, BDGDP, $r)$ & $\begin{array}{c}-0.99 \\
(-1.54)\end{array}$ & $\begin{array}{l}5.52^{* *} \\
(3.52)\end{array}$ & $\begin{array}{l}0.78^{\star *} \\
(3.54)\end{array}$ & $\begin{array}{c}0.08 \\
(0.52)\end{array}$ & & & 1991 & -4.84 & 0.48 & 1.00 \\
\hline RS: $C A G D P=f(C, B D G D P, r)$ & $\begin{array}{c}0.66 \\
(0.83) \\
\end{array}$ & $\begin{array}{l}7.72^{*} \\
(2.22)\end{array}$ & $\begin{array}{l}1.27^{\star *} \\
(4.60)\end{array}$ & $\begin{array}{c}0.43 \\
(1.97) \\
\end{array}$ & $\begin{array}{l}-1.78^{* *} \\
(-4.26) \\
\end{array}$ & $\begin{array}{l}-1.69^{\star *} \\
(-2.73)\end{array}$ & 1985 & $-5.89^{*}$ & 0.57 & 1.06 \\
\hline \multicolumn{11}{|c|}{ Portugal:1970-1998: One regressor } \\
\hline SC: $C A G D P=f(C, B D G D P)$ & $\begin{array}{c}-0.60 \\
(-0.39) \\
\end{array}$ & & $\begin{array}{c}0.27 \\
(1.35) \\
\end{array}$ & & & & & -2.46 & 0.06 & 0.80 \\
\hline LS: CAGDP=f(C, BDGDP) & $\begin{array}{c}0.94 \\
(0.54)\end{array}$ & $\begin{array}{c}-3.66 \\
(-1.67) \\
\end{array}$ & $\begin{array}{c}0.41 \\
(1.95) \\
\end{array}$ & & & & 1994 & -3.03 & 0.15 & 0.90 \\
\hline RS: CAGDP=f(C, BDGDP) & $\begin{array}{c}2.47 \\
(1.48)\end{array}$ & $\begin{array}{l}-6.90^{\star \star} \\
(-3.01)\end{array}$ & $\begin{array}{l}0.82^{\star *} \\
(4.09)\end{array}$ & & $\begin{array}{c}-1.39^{\star *} \\
(-4.54)\end{array}$ & & 1985 & $-4.86^{*}$ & 0.52 & 1.60 \\
\hline \multicolumn{11}{|c|}{ Portugal:1970-1998: Two regressors } \\
\hline SC: $C A G D P=f(C, B D G D P, r)$ & $\begin{array}{c}-0.60 \\
(-0.41)\end{array}$ & & $\begin{array}{c}0.23 \\
(1.20)\end{array}$ & $\begin{array}{c}0.19 \\
(1.74)\end{array}$ & & & & -2.31 & 0.16 & 0.73 \\
\hline LS: CAGDP=f(C, BDGDP, r $)$ & $\begin{array}{c}2.46 \\
(1.27)\end{array}$ & $\begin{array}{l}-4.70^{*} \\
(-2.25) \\
\end{array}$ & $\begin{array}{c}0.45^{*} \\
(2.19)\end{array}$ & $\begin{array}{l}0.35^{\star *} \\
(2.83)\end{array}$ & & & 1990 & $-5.36^{*}$ & 0.30 & 1.00 \\
\hline RS: CAGDP=f(C, BDGDP, $r)$ & $\begin{array}{c}3.60 \\
(1.92) \\
\end{array}$ & $\begin{array}{c}-11.33^{* *} \\
(-3.19)\end{array}$ & $\begin{array}{l}0.59^{* *} \\
(2.96)\end{array}$ & $\begin{array}{l}0.37^{* *} \\
(2.95)\end{array}$ & $\begin{array}{l}-1.60^{*} \\
(-2.40) \\
\end{array}$ & $\begin{array}{c}-0.06 \\
(-0.17) \\
\end{array}$ & 1990 & -5.07 & 0.44 & 1.08 \\
\hline
\end{tabular}


Table B2 continued

\begin{tabular}{|c|c|c|c|c|c|c|c|c|c|c|}
\hline $\begin{array}{l}\text { Country, sample periods } \\
\text { and model types }\end{array}$ & $\mu_{1}$ & $\mu_{1} \varphi_{1 \tau}$ & $\beta_{1}$ & $\beta_{2}$ & $\beta_{3} \varphi_{1 \tau}$ & $\beta_{4} \varphi_{1 \tau}$ & $\begin{array}{l}\text { Break } \\
\text { year }\end{array}$ & ADF & $\mathrm{R}^{2}$ & DW \\
\hline \multicolumn{11}{|c|}{ Spain:1962-1998: One regressor } \\
\hline SC: $C A G D P=f(C, B D G D P)$ & $\begin{array}{l}-1.23^{*} \\
(-2.53)\end{array}$ & & $\begin{array}{c}-0.07 \\
(-0.56)\end{array}$ & & & & & $-5.45^{\star *}$ & 0.01 & 0.77 \\
\hline LS: CAGDP=f(C, BDGDP) & $\begin{array}{c}-0.89 \\
(-1.70) \\
\end{array}$ & $\begin{array}{l}-1.12 \\
(-1.59) \\
\end{array}$ & $\begin{array}{c}-0.20 \\
(-1.34) \\
\end{array}$ & & & & 1975 & $-5.79^{* *}$ & 0.08 & 0.85 \\
\hline RS: CAGDP=f(C, BDGDP) & $\begin{array}{l}-0.33 \\
(-0.33) \\
\end{array}$ & $\begin{array}{c}-1.82 \\
(-1.38)\end{array}$ & $\begin{array}{c}0.31 \\
(0.55)\end{array}$ & & $\begin{array}{l}-0.53 \\
(-0.91)\end{array}$ & & 1980 & $-6.00^{\star *}$ & 0.07 & 0.82 \\
\hline \multicolumn{11}{|c|}{ Spain:1962-1998: Two regressors } \\
\hline SC: $C A G D P=f(C, B D G D P, r)$ & $\begin{array}{l}-1.26^{*} \\
(-2.45) \\
\end{array}$ & & $\begin{array}{c}-0.08 \\
(-0.61)\end{array}$ & $\begin{array}{c}-0.01 \\
(-0.25) \\
\end{array}$ & & & & $-5.44^{\star \star}$ & 0.01 & 0.77 \\
\hline LS: CAGDP=f(C, BDGDP, r $)$ & $\begin{array}{l}-0.90 \\
(-1.61) \\
\end{array}$ & $\begin{array}{c}-1.11 \\
(-1.55)\end{array}$ & $\begin{array}{l}-0.20 \\
(-1.31) \\
\end{array}$ & $\begin{array}{l}-0.004 \\
(-0.07)\end{array}$ & & & 1975 & $-5.78^{\star *}$ & 0.08 & 0.85 \\
\hline RS: $C A G D P=f(C, B D G D P, r)$ & $\begin{array}{c}-0.61 \\
(-1.12) \\
\end{array}$ & $\begin{array}{c}1.32 \\
(0.73) \\
\end{array}$ & $\begin{array}{l}-0.004 \\
(-0.03) \\
\end{array}$ & $\begin{array}{c}0.07 \\
(1.36)\end{array}$ & $\begin{array}{c}-0.10 \\
(-0.33)\end{array}$ & $\begin{array}{l}-0.65^{\star \star} \\
(-2.85)\end{array}$ & 1989 & $-6.45^{\star *}$ & 0.28 & 1.16 \\
\hline \multicolumn{11}{|c|}{ Sweden:1960-2000: One regressor } \\
\hline SC: $\mathrm{CAGDP}=\mathrm{f}(\mathrm{C}, \mathrm{BDGDP})$ & $\begin{array}{c}0.22 \\
(0.61)\end{array}$ & & $\begin{array}{c}0.14^{*} \\
(2.06)\end{array}$ & & & & & -2.30 & 0.10 & 0.53 \\
\hline LS: CAGDP=f(C, BDGDP) & $\begin{array}{l}-0.40 \\
(-1.56)\end{array}$ & $\begin{array}{l}3.57^{\star \star} \\
(6.88)\end{array}$ & $\begin{array}{l}0.17^{\star *} \\
(3.66)\end{array}$ & & & & 1993 & -4.14 & 0.60 & 1.04 \\
\hline RS: CAGDP=f(C, BDGDP) & $\begin{array}{c}-0.46 \\
(-1.61) \\
\end{array}$ & $\begin{array}{l}3.71^{*} \\
(6.20)\end{array}$ & $\begin{array}{l}0.15^{*} \\
(2.29) \\
\end{array}$ & & $\begin{array}{c}0.05 \\
(0.48) \\
\end{array}$ & & 1993 & -4.22 & 0.60 & 1.04 \\
\hline \multicolumn{11}{|c|}{ Sweden:1960-2000: Two regressors } \\
\hline SC: $C A G D P=f(C, B D G D P, r)$ & $\begin{array}{c}0.09 \\
(0.26)\end{array}$ & & $\begin{array}{l}0.14^{*} \\
(2.06)\end{array}$ & $\begin{array}{c}0.14 \\
(1.18) \\
\end{array}$ & & & & -2.63 & 0.13 & 0.65 \\
\hline LS: CAGDP=f(C, BDGDP, $r)$ & $\begin{array}{l}-0.27 \\
(-0.75)\end{array}$ & $\begin{array}{l}1.87^{\star \star} \\
(2.78)\end{array}$ & $\begin{array}{l}0.16^{*} \\
(2.43)\end{array}$ & $\begin{array}{c}0.02 \\
(0.22)\end{array}$ & & & 1990 & -4.22 & 0.28 & 0.74 \\
\hline RS: $C A G D P=f(C, B D G D P, r)$ & $\begin{array}{l}-0.40 \\
(-1.05) \\
\end{array}$ & $\begin{array}{l}2.71^{* *} \\
(3.10)\end{array}$ & $\begin{array}{c}0.14 \\
(1.58)\end{array}$ & $\begin{array}{c}0.01 \\
(0.04) \\
\end{array}$ & $\begin{array}{c}0.07 \\
(0.54) \\
\end{array}$ & $\begin{array}{c}-0.02 \\
(-0.07) \\
\end{array}$ & 1991 & -4.08 & 0.38 & 0.81 \\
\hline \multicolumn{11}{|c|}{ Switzerland:1960-2000: One regressor } \\
\hline SC: $\mathrm{CAGDP}=\mathrm{f}(\mathrm{C}, \mathrm{BDGDP})$ & $\begin{array}{l}3.25^{\star *} \\
(5.27)\end{array}$ & & $\begin{array}{c}-1.29 \\
(-1.86)\end{array}$ & & & & & 0.49 & 0.08 & 0.26 \\
\hline LS: CAGDP=f(C, BDGDP) & $\begin{array}{l}1.67^{* *} \\
(3.03)\end{array}$ & $\begin{array}{l}5.15^{\star *} \\
(5.48)\end{array}$ & $\begin{array}{c}-0.74 \\
(-1.38) \\
\end{array}$ & & & & 1987 & -3.04 & 0.49 & 0.50 \\
\hline RS: CAGDP=f(C, BDGDP) & $\begin{array}{l}1.58^{* *} \\
(2.83)\end{array}$ & $\begin{array}{l}5.50^{* *} \\
(5.34)\end{array}$ & $\begin{array}{l}-1.15 \\
(-1.60) \\
\end{array}$ & & $\begin{array}{c}0.93 \\
(0.86)\end{array}$ & & 1987 & -3.01 & 0.49 & 0.58 \\
\hline \multicolumn{11}{|c|}{ Switzerland:1960-2000: Two regressors } \\
\hline SC: $C A G D P=f(C, B D G D P, r)$ & $\begin{array}{l}3.25^{* *} \\
(6.38)\end{array}$ & & $\begin{array}{c}-1.85^{\star *} \\
(-3.15)\end{array}$ & $\begin{array}{l}1.21^{\star \star} \\
(4.40)\end{array}$ & & & & -1.55 & 0.39 & 0.56 \\
\hline LS: CAGDP=f(C, BDGDP, $r)$ & $\begin{array}{l}2.50^{* *} \\
(5.85)\end{array}$ & $\begin{array}{c}5.26 \\
(4.97)\end{array}$ & $\begin{array}{c}-1.22 \\
(-2.57)\end{array}$ & $\begin{array}{c}0.90 \\
(3.97)\end{array}$ & & & 1994 & -3.02 & 0.63 & 0.88 \\
\hline RS: CAGDP=f(C, BDGDP, r $)$ & $\begin{array}{l}2.29^{\star *} \\
(5.05) \\
\end{array}$ & $\begin{array}{l}6.48^{* *} \\
(4.94) \\
\end{array}$ & $\begin{array}{c}-1.97^{\star *} \\
(-2.97)\end{array}$ & $\begin{array}{l}1.01^{* *} \\
(3.85)\end{array}$ & $\begin{array}{l}2.72^{*} \\
(2.60) \\
\end{array}$ & $\begin{array}{c}-1.26 \\
(-1.45) \\
\end{array}$ & 1990 & -4.44 & 0.65 & 0.89 \\
\hline
\end{tabular}


Table B2 continued

\begin{tabular}{|c|c|c|c|c|c|c|c|c|c|c|}
\hline $\begin{array}{l}\text { Country, sample periods } \\
\text { and model types }\end{array}$ & $\mu_{1}$ & $\mu_{1} \varphi_{1 \tau}$ & $\beta_{1}$ & $\beta_{2}$ & $\beta_{3} \varphi_{1 \tau}$ & $\beta_{4} \varphi_{1 \tau}$ & $\begin{array}{c}\text { Break } \\
\text { year }\end{array}$ & ADF & $\mathrm{R}^{2}$ & DW \\
\hline \multicolumn{11}{|c|}{ United Kingdom: 1960-1999: One regressor } \\
\hline SC: $\mathrm{CAGDP}=\mathrm{f}(\mathrm{C}, \mathrm{BDGDP})$ & $\begin{array}{c}-0.87^{\star *} \\
(-2.30)\end{array}$ & & $\begin{array}{c}-0.08 \\
(-0.76)\end{array}$ & & & & & -3.01 & 0.01 & 0.52 \\
\hline LS: CAGDP=f(C, BDGDP) & $\begin{array}{c}-0.08 \\
(-0.21) \\
\end{array}$ & $\begin{array}{c}-1.87^{* *} \\
(-3.98) \\
\end{array}$ & $\begin{array}{c}-0.04 \\
(-0.49) \\
\end{array}$ & & & & 1985 & -4.11 & 0.31 & 0.80 \\
\hline RS: CAGDP=f(C, BDGDP) & $\begin{array}{c}-0.23 \\
(-0.57)\end{array}$ & $\begin{array}{l}-2.22^{* *} \\
(-3.02)\end{array}$ & $\begin{array}{c}-0.02 \\
(-0.13)\end{array}$ & & $\begin{array}{c}-0.19 \\
(-0.96)\end{array}$ & & 1989 & -3.69 & 0.25 & 0.72 \\
\hline \multicolumn{11}{|c|}{ United Kingdom: 1960-1999: Two regressors } \\
\hline SC: $C A G D P=f(C, B D G D P, r)$ & $\begin{array}{l}-0.81^{*} \\
(2.07)\end{array}$ & & $\begin{array}{c}-0.06 \\
(-0.49)\end{array}$ & $\begin{array}{c}-0.04 \\
(-0.58)\end{array}$ & & & & -3.05 & 0.02 & 0.53 \\
\hline LS: CAGDP=f(C, BDGDP, $r)$ & $\begin{array}{c}-0.05 \\
(-0.14) \\
\end{array}$ & $\begin{array}{l}-2.26^{\star \star} \\
(-4.25)\end{array}$ & $\begin{array}{c}-0.10 \\
(-0.99) \\
\end{array}$ & $\begin{array}{c}0.09 \\
(1.50)\end{array}$ & & & 1985 & -4.39 & 0.35 & 0.94 \\
\hline RS: $C A G D P=f(C, B D G D P, r)$ & $\begin{array}{c}0.36 \\
(0.86)\end{array}$ & $\begin{array}{l}-2.72^{\star *} \\
(-3.04)\end{array}$ & $\begin{array}{c}0.07 \\
(0.55)\end{array}$ & $\begin{array}{c}0.07 \\
(1.10)\end{array}$ & $\begin{array}{c}-0.34 \\
(-1.80)\end{array}$ & $\begin{array}{c}-0.09 \\
(-0.44)\end{array}$ & 1985 & -4.51 & 0.41 & 1.00 \\
\hline \multicolumn{11}{|c|}{ USA:1960-2000: One regressor } \\
\hline SC: $C A G D P=f(C, B D G D P)$ & $\begin{array}{c}-0.79^{*} \\
(-2.51) \\
\end{array}$ & & $\begin{array}{c}0.02 \\
(0.21) \\
\end{array}$ & & & & & -0.27 & 0.001 & 0.21 \\
\hline LS: CAGDP=f(C, BDGDP) & $\begin{array}{c}-0.08 \\
(-0.36)\end{array}$ & $\begin{array}{l}-2.22^{\star \star} \\
(-7.19)\end{array}$ & $\begin{array}{c}-0.19^{*} \\
(-2.39)\end{array}$ & & & & 1980 & -3.92 & 0.58 & 0.58 \\
\hline RS: CAGDP=f(C, BDGDP) & $\begin{array}{c}0.16 \\
(0.47)\end{array}$ & $\begin{array}{c}-2.41^{* *} \\
(-5.19)\end{array}$ & $\begin{array}{c}-0.01 \\
(-0.05)\end{array}$ & & $\begin{array}{c}-0.19 \\
(-0.91)\end{array}$ & & 1979 & -4.40 & 0.54 & 0.64 \\
\hline \multicolumn{11}{|c|}{ USA:1960-2000: Two regressors } \\
\hline SC: $C A G D P=f(C, B D G D P, r)$ & $\begin{array}{c}-0.17 \\
(-0.54)\end{array}$ & & $\begin{array}{c}0.02 \\
(0.27)\end{array}$ & $\begin{array}{l}-0.41^{* *} \\
(-3.68)\end{array}$ & & & & -1.26 & 0.26 & 0.40 \\
\hline LS: CAGDP=f(C, BDGDP, r $)$ & $\begin{array}{c}0.28 \\
(1.12)\end{array}$ & $\begin{array}{c}-1.84^{\star *} \\
(-5.53)\end{array}$ & $\begin{array}{c}-0.15 \\
(-1.82) \\
\end{array}$ & $\begin{array}{l}-0.23^{*} \\
(-2.52) \\
\end{array}$ & & & 1977 & -3.98 & 0.60 & 0.50 \\
\hline RS: $C A G D P=f(C, B D G D P, r)$ & $\begin{array}{c}0.12 \\
(0.34)\end{array}$ & $\begin{array}{l}-1.52^{\star \star} \\
(-3.02)\end{array}$ & $\begin{array}{c}-0.02 \\
(-0.14) \\
\end{array}$ & $\begin{array}{c}0.02 \\
(0.13)\end{array}$ & $\begin{array}{c}-0.20 \\
(-1.03) \\
\end{array}$ & $\begin{array}{l}-0.48^{\star \star} \\
(-2.81)\end{array}$ & 1980 & -4.78 & 0.72 & 0.76 \\
\hline
\end{tabular}

Critical values $(\mathrm{CVs})$ with one regressor and without structural breaks (nobs $=40)$ : -3.64 (at 5\% level of significance); -3.29 (at 10\% level of significance)

CVs with two regressors and without structural breaks ((nobs=40?)): - 4.07 (at 5\% level of significance); -3.71 (at 10\% level of significance)

Critical values with one regressor and structural breaks : at 5\% level of significance: LS $=4.61$, $\mathrm{LST}=-4.99, \mathrm{RS}=-4.95:$ at $10 \%$ level of significance: $\mathrm{LS}=-4.34, \mathrm{LST}=-4.72, \mathrm{RS}=-4.68$

Critical values with two regressor and structural breaks : at $5 \%$ level of significance: $\mathrm{LS}=-4.92$, $\mathrm{LST}=-5.29, \mathrm{RS}=-5.50:$ at $10 \%$ level of significance: $\mathrm{LS}=-4.69, \mathrm{LST}=-5.03, \mathrm{RS}=-5.23$

** - significant at the $1 \%$ level, * - significant at the $5 \%$ level

Shift types are LS: Level Shift (GHp103 cagdpll this cagdpse "C"); LST: Level Shift with Trend (GHp103 cagdpll this cagdpse "C/T"), RS: Regime Shift (GHp103 cagdpll this cagdpse "C/S") 